Department of Econometrics and Business Statistics

http://business.monash.edu/econometrics-and-business-

$$
\text { statistics/research/publications }
$$

\title{
Local logit regression for recovery rate
}

Nithi Sopitpongstorn, Param Silvapulle and Jiti Gao 


\title{
Local logit regression for recovery rate
}

\author{
Nithi Sopitpongstorn Param Silvapulle Jiti Gao \\ Monash Business School
}

Department of Econometrics and Business Statistics

Monash University

\begin{abstract}
We propose a flexible and robust nonparametric local logit regression for modelling and predicting defaulted loans' recovery rates that lie in $[0,1]$. Applying the model to the widely studied Moody's recovery dataset and estimating it by a data-driven method, the local logit regression uncovers the underlying nonlinear relationship between the recovery and covariates, which include loan/borrower characteristics and economic conditions. We find some significant nonlinear marginal and interaction effects of conditioning variables on recoveries of defaulted loans. The presence of such nonlinear economic effects enriches the local logit model specification that supports the improved recovery prediction. This paper is the first to study a nonparametric regression model that not only generates unbiased and improved recovery predictions of defaulted loans relative to the parametric counterpart, it also facilitates reliable inference on marginal and interaction effects of loan/borrower characteristics and economic conditions. Moreover, incorporating these nonlinear marginal and interaction effects, we improve the specification of parametric regression for fractional response variable, which we call "calibrated" model, the predictive performance of which is comparable to that of local logit model. This calibrated parametric model will be attractive to applied researchers and industry professionals working in the risk management area and unfamiliar with nonparametric machinery.
\end{abstract}

Keywords: Loss given default, credit risk, nonlinearity, kernel estimation, defaulted debt, simulation

JEL Classifications: C14, C53, G02, G32 


\section{Introduction}

The recovery of debt in the event of default is a crucial determinant of the default risk premium required by a lender and the regulatory capital charged to minimize exposure to losses. Basel II and III offer regulatory incentives to internationally active financial institutions for the development of an internal advanced measurement approach to computing capital to be held against credit risk ${ }^{1}$ exposure (BIS, 2004). Furthermore, the pricing of default risk insurance and the advent of distressed debt as an investment class provide further incentives for improved understanding of the distribution of recoveries of loans in the event of default. Recognizing the importance of capturing the typical features of recovery distribution and regression modelling of recoveries on conditioning variables, such as loan/borrower characteristics and economic conditions at the time of defaults, recent years have witnessed a notable increase in research investigation into modelling recovery rate mostly for the purpose of recovery prediction by academics and industry professionals.

In the quest for finding a model for the recovery rate relating to conditioning variables, several studies observed that this modelling exercise presents some challenges due to the key empirical features of recovery rates: (i) it is continuous, fractional \& bounded in $[0,1]$; (ii) its empirical density is bimodal, asymmetry with high proportions of recoveries at the boundaries zero and one ${ }^{2}$; (iii) in the presence of observations at 0 and 1 , trimming and transformation, and back-transformation of recoveries are needed for the use of valid statistical theory. Such transformation introduces bias in the model estimates, resulting in unreliable statistical inference, more on this discussed later in this section; and (iv) despite the growing body of evidence of the presence of nonlinearity in the recovery-covariate relatioship, little attempt has been made in the literature to improve the specification of the widely used linear regression model so that the nonlinearity in the relationship is transparent and the key determinants of recovery can be found. ${ }^{3}$

The documented empirical features of historical recovery rates suggest the need to be

\footnotetext{
${ }^{1}$ The three components that constitute credit risk include probability of default, recovery rate and exposure at default. Recovery rate $=(1$ - loss given default $)$.

${ }^{2}$ See Schuermann (2004); Bastos (2010); Calabrese and Zenga (2010); Tong, Mues, and Thomas (2013) for details.

${ }^{3}$ See Sopitpongstorn, Gao, Silvapulle, and Zhang (2014, 2016); Yao, Crook, and Andreeva (2015); Loterman, Brown, Martens, Mues, and Baesens (2012); Qi and Zhao (2011) for discussions on nonlinear approaches for recovery modelling.
} 
prudent in applying popular parametric models, such as OLS regression and calibrated Beta distributions for statistical inference. The OLS regression model is simple with the normality assumption, which would not capture the above typical features of recovery distribution. Despite Beta distributions offering a simple, parsimonious way of capturing a very broad range of distributional shapes over the unit interval, De Servigny, Renault, and de Servigny (2004); Sopitpongstorn et al. (2014, 2016) observe that they cannot accommodate bi-modality, or probability masses near zero and unity - key features of empirical recovery distribution.

There exists a vast literature on the parametric or semiparametric regression for modelling recovery rate with the main focus on improving the predictive ability of the regression models; see, Gupton and Stein (2005); Bastos (2010); Qi and Yang (2009); Altman and Kalotay (2014), among others. As recovery lies in $[0,1]$ with nonzero masses at 0 and 1 , the recoveries are trimmed at the boundaries and then the data in unit interval is transformed to real line for valid statistical modeling. Such a transformation introduces bias to the model estimates, resulting in unreliable statistical inference and the recovery rate $(\mathrm{RR})$ prediction. To see how the bias arises, consider the several steps involved in this process. 1) Trim both boundaries with an arbitrary value $\nu$ so that the recovery rate range is $(0,1) ; 2)$ Transform $(0,1)$ to $(-\infty, \infty)$ using a monotonic function $\Phi(\cdot)$ such as inverse Gaussian, Beta, and logistic function; 3) Regress the transformed RR, say $R R^{\nu}$ on the set of conditioning variables; and 4) Apply the inverse transformation $\Phi^{-1}(\cdot)$ to predicted $R R^{\nu}$ back to the original range. However, such a transformation process introduces bias to the model estimates because $\Phi\left(E\left(Y^{(\nu)} \mid x\right)\right) \neq E\left(\Phi\left(Y^{(\nu)}\right) \mid x\right)$. The studies that used such transformation appeared to have relaxed this inequality and overlooked the presence of bias in the model estimates. An exception is the $\mathrm{QMLE}^{4}$ regression developed by Papke and Wooldridge (1996) specifically for fractional data. Thus, the aforementioned bias would not arise in this model. Several studies have applied the linear QMLE regression to recovery rate and found that the model provides $\mathrm{RR}$ better prediction than the other regression models (Dermine \& De Carvalho, 2006; Khieu, Mullineaux, \& Yi, 2012). Furthermore, Qi and Yang (2009), and Sopitpongstorn et al. (2014) showed that the QMLE-regression for fractional data also has better out-of-sample recovery predictive accuracy than the alternative parametric regressions that have been popular in the

\footnotetext{
${ }^{4}$ Quasi maximum likelihood estimation.
} 
recovery modelling literature.

There are several studies investigated the predictive performance of non-parametric models such as neural networks relative to some parametric regression specifications. Using the US data of defaulted loans and bonds, Qi and Zhao (2011) and others demonstrated that recovery predictions based on regression trees and neural networks outperform those of parametric regression models. They attribute the success of non-parametric models mostly to their ability to accommodate some non-linear associations between recoveries and the conditioning variables. Moreover, in establishing the predictive ability of nonparametric techniques relative to parametric regression models, the studies by Bastos (2010) and Qi and Zhao (2011) highlight the potential weaknesses of these approaches. They acknowledge that neural networks are "black-box" models which do not provide any transparent recovery-covariate relationships that strengthen the predictions. Despite the regression trees being more transparent and intuitive, they can become unmanageable in size and include recovery-covariate relationships that are difficult to reconcile with priori expectations. See Bastos (2010) and Qi and Zhao (2011), among others for more discussion on regression trees. Recently, Altman and Kalotay (2014) have developed a semiparametric mixture distribution model in that they adopt a Bayesian perspective and model the distribution of recoveries using mixtures of Gaussian distributions. By taking the appropriate probability weighted average of Gaussian components, they accommodate various features of recovery distribution. Additionally, the ordered probit regression specification accommodates some non-linearity in the relation between continuous conditioning variables and recovery outcomes.

In analysing the economic effects of covariates, the collateralization, the degree of subordination and debt cushion ${ }^{5}$ are found to be the key determinants of recovery of defaulted loan. Additionally, the larger the debt cushion, the higher the expected recovery of defaulted loan; see Van de Castle and Keisman (1999) for details. Also, as expected recoveries are found to be lower during economic downturns. The analysis of Altman and Kalotay (2014) highlights some nonlinear marginal effects of some covariates.

Clearly, our discussion in the previous paragraphs and the growing weight of empirical evidence uncover much about the important influences on debt recovery outcomes, as well

\footnotetext{
${ }^{5}$ The proportional value of claims subordinate to the debt at a given seniority is known as the debt cushion (Altman and Kalotay (2014)).
} 
as highlighting the problems and challenges intrinsic to building statistical recovery models to account for defaulted loan/borrower characteristics and macroeconomic conditions at the time of default and to capture the specific features of recovery distributions. In this paper, we build on insights from the findings of huge empirical research as well as studies documenting the merits of non-parametric and semiparametric approaches and the regression for fractional data for recovery predictions and marginal effect analysis, we propose a flexible and robust nonparametric local logit model for recovery rates of defaulted loans.

Our paper makes several principal contributions, which will highlight the novelty of our proposed local logit model mostly for its flexibility in accommodating nonlinear recoverycovariate relationships, and thus enriching the model specification which supports the improved recovery prediction. First, our proposed local logit model has a flexible model specification in that the unknown coefficients are assumed to be functions of all covariates. The data-driven kernel estimation method will uncover the underlying nonlinear recoverycovariate relationship, which facilitates the analysis of the marginal and interaction effects of the conditioning variables on recoveries, which will be demonstrated in our empirical application presented in this paper.

Second, the local logit model estimates are robust to various shapes and features of recovery distribution ${ }^{6}$ discussed in the previous paragraphs, providing reliable statistical inference. Third, our model is developed specifically for fractional data. To propose the local logit model for fractional data, we integrate the ideas presented in Papke and Wooldridge (1996) who introduced the QMLE regression for fractional data and Frölich (2006) who developed local logit model for binary discrete variables and demonstrated its superiority to parametric counterparts. Thus, there is no need for trimming and transforming recoveries for regression modelling. As a result, the aforementioned bias will not arise in our model, improving further the reliability of statistical inference and recovery prediction.

Fourth, we apply the local logit regression to the widely studied Moody's recovery rate dataset spanning 18 years, and we demonstrate that the ways in which the loan/borrower characteristics and economic conditions at the time of defaults and their interactions influence the recoveries of defaulted loans and their predictions. We provide a comprehensive

\footnotetext{
${ }^{6}$ In this paper, we provide simulation study to clarify this robustness.
} 
analysis of nonlinear marginal and interactions effects on recoveries, whereas the main focus of previous studies has been on the prediction of recoveries and linear marginal effects. Recently, Altman and Kalotay (2014) estimated the nonlinear marginal effects of continuous variables on recoveries. Our model would not only capture the nonlinearity in the marginal effects of debt cushion and stress index, it will also accommodate nonlinear interactions between continuous and discrete variables. Additionally, our modelling process does not require the trimming and transformation of recoveries, whereas such transformation is needed in their semiparametric model similar to many regression based models for recoveries studied in the literature.

The remainder of this paper is organized as follows. In the next section, the nonparametric local logit regression for $[0,1]$ bounded response data is proposed along with the estimation method, followed by a brief discussion of the parametric QMLE-regression for fractional data and the estimation method. Section 3 conducts a simulation study to assess various properties and the robustness of the proposed model and analyses the results. Section 4 provides a specification test. Section 5 briefly discusses the Moody's data and reports some results of the preliminary analysis. Section 6 conducts the empirical analysis and assesses the out-of-sample recovery predictability of the models. Section 7 concludes this paper. The simulations results are reported in the Appendix A. The empirical results are reported in the Appendix B.

\section{Methodology}

In this section, we discuss the parametric QMLE regression for fractional response variable (QMLE-RFRV) and propose a nonparametric local logit model and the estimation methods which include the choice of kernel functions and bandwidth selection criterion. Furthermore, we briefly discuss several criteria in order to evaluate the predictive performance of the proposed model relative to the parametric counterpart. 


\subsection{Parametric regression for $[0,1]$ bounded data}

The parametric QMLE-RFRV is the theoretically valid model for the fractional response variable, such as the recovery rate $(\mathrm{RR})$. The conditional mean is given as:

$$
E(Y \mid X=x)=\Lambda\left(x^{\prime} \gamma\right)
$$

where $Y$ is the continuous $[0,1]$ bounded variable (i,e. $0 \leq Y \leq 1), X$ is the vector of $k$ covariates (which is individual loan characteristics - a mixture of continuous and discrete variables in the empirical example), $\Lambda(\cdot)$ is the logistic function, $0<\Lambda(\cdot)<1$, and $\gamma$ is a vector of unknown parameters. Papke and Wooldridge (1996) proposed a quasi-maximum likelihood estimation (QMLE) method. The unknown vector of parameters are estimated as:

$$
\hat{\gamma}=\arg \max _{\gamma} \sum_{i=1}^{n} Y_{i} \log \left(\Lambda\left(X_{i}^{\prime} \gamma\right)\right)+\left(1-Y_{i}\right) \log \left(1-\Lambda\left(X_{i}^{\prime} \gamma\right)\right)
$$

The estimator in (2) is consistent and asymptotically normal, these properties being robust to various conditional distributional assumptions.

The main assumption of the QMLE-RFRV is the correctly specified functional form for the conditional mean. However, the conditional mean of this model can be misspecified in practice because the underlying correct functional form is largely unknown. We want to improve the specification of the conditional mean of QMLE-RFRV, which might include sufficient number of interaction terms, polynomials and discretized continuous variables and so on, by exploiting information provided by the estimates of local logit model. The calibrated QMLE-RFRV is presented in Section 5.

\section{$2.2 \quad$ Local logit regression}

This study proposes a local logit regression for fractional response variable and a data driven nonparametric method to estimate the model. As will be seen, the local logit model is flexible to accommodate the underlying any complex nonlinear relationship between $\mathrm{RR}$ and covariates. The conditional mean is defined as: 


$$
E(Y \mid X=x)=\Lambda\left(x^{\prime} \beta(x)\right)
$$

where $x=\left(x_{1}, . ., x_{k}\right)^{\prime}$ is $k \times 1$ vector, $\beta(x)$ is a vector of unknown local logit estimator is the function of $x$.

We obtain the estimators of local logit model by maximizing the local likelihood function as:

$$
\hat{\beta}(x)=\arg \max _{\beta(x)} \sum_{i=1}^{n} Y_{i} \log \left(\Lambda\left(X_{i}^{\prime} \beta(x)\right)\right)+\left(1-Y_{i}\right) \log \left(1-\Lambda\left(X_{i}^{\prime} \beta(x)\right)\right) \mathbb{K}_{H}\left(X_{i}, x\right),
$$

where $\mathbb{K}_{H}\left(X_{i}, x\right)$ is a product of $k$ kernel functions associated with $\left(x_{1}, \ldots, x_{k}\right)$ for a given a vector of bandwidths $H=\left(h_{1}, \ldots, h_{k}\right)^{\prime}$. The local logit model parameter $\beta(x)$ - which is a function of covariates $x$ - is locally estimated based on a kernel weights $\mathbb{K}_{H}\left(X_{i}, x\right)$, which determine the local distance between $X_{i}$ and a specified value of vector $x$ for a given set of bandwidths $H$.

Our study employs two different kernel functions: a Gaussian kernel function for continuous variables and a kernel function which is constructed specifically for categorical variables. Let us define:

$$
X_{i}=\left(X_{i}^{c}, X_{i}^{d}\right)
$$

where the continuous regressors with $p$ dimensions is $X_{i}^{c} \in \mathbf{R}^{p}$, the remaining regressors $X_{i}^{d}$ is a $q \times 1$ vector of categorical variables, and $p+q=k$. For any $t^{t h}$ component in $X_{i}^{d}$, where $t \in\{1, \ldots, q\}$, each component can take a discrete value such as $X_{t, i}^{d} \in\left\{0,1, \ldots, c_{t}-1\right\}$, where $c_{t} \geq 2$ is the number of categories of $X_{t, i}^{d}$. Clearly, $c_{t}=2$ for the dummy variable. In what follows, the two kernel functions that we use in the estimation of local logit are defined.

\section{A kernel function for continuous variable}

The standard Gaussian kernel function is employed for any continuous variable $\left(X_{i}^{c}\right)$ which is defined as:

$$
\kappa_{s}\left(X_{s, i}^{c}, x_{s}^{c}, h_{s}\right)=\frac{1}{\sqrt{2 \pi}} \exp \left(-\frac{1}{2}\left(\frac{X_{s, i}^{c}-x_{s}^{c}}{h_{s}}\right)^{2}\right)
$$


where $s=1, . ., p, \kappa(\cdot)$ is the Gaussian kernel function, and $h_{s}$ is a bandwidth associated with $s^{\text {th }}$ continuous variable.

\section{A kernel function for discrete variable}

For the discrete variable, we apply the kernel function proposed by Racine and Li (2004) which is defined as:

$$
\lambda_{t}\left(X_{t, i}^{d}, x_{t}^{d}, l_{t}\right)= \begin{cases}1, & \text { if } X_{t, i}^{d}=x_{t}^{d} \\ l_{t}, & \text { otherwise }\end{cases}
$$

where we assume that the $t^{\text {th }}$ categorical variable, $l_{t}$ is the bandwidth associated with $\lambda_{t}(\cdot)$, and $0<l_{t} \leq 1$.

The product of the kernel functions ${ }^{7}$ in (5) and (6) are functions of bandwidths and the optimum selection of which are crucial in the estimation of the local logit model. There are several bandwidth selection methods available for the non-parametric estimation. Although the plug-in method is popular, its application is limited as it does not work well in the small sample setting and the high dimensional independent variables $x$. This study use, on the other hand, the least-squares cross-validation which is commonly applied in practice to select the bandwidth that minimize a certain loss function.

In this study, we select the set of bandwidths $H=\left(h_{1}, . ., h_{p}, l_{1}, . ., l_{q}\right)$ that minimizes an objective function, which is the sum of prediction error squares, defined as:

$$
C V=\sum_{i=1}^{n}\left(Y_{i}-\Lambda\left(X_{i}^{\prime} \hat{\beta}\left(x_{-i} \mid H\right)\right)\right)^{2}
$$

where $\hat{\beta}\left(x_{-i} \mid H\right)$ is a $k \times 1$ vector of leave-one-out estimates of local logit estimators associated with $x_{i}$ which is a solution to:

$$
\arg \max _{\beta\left(X_{i}\right)} \sum_{j=1, i \neq j}^{n} Y_{j} \log \left(\Lambda\left(X_{j}^{\prime} \beta\left(X_{i}\right)\right)\right)+\left(1-Y_{j}\right) \log \left(1-\Lambda\left(X_{j}^{\prime} \beta\left(X_{i}\right)\right)\right) \mathbb{K}_{H}\left(X_{j}, X_{i}\right)
$$

It is worth noting that the optimal bandwidth in (7) would be very large if the unknown underlying functional form is indeed the standard linear function, $\Lambda\left(X^{\prime} \gamma\right)$. When the sizes of all bandwidths increase as $n$ goes to infinity, the equal kernel weights are assigned for all

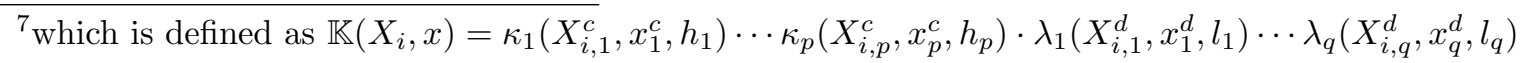


$i$. Specifically, the large bandwidths would cause the product of kernel functions in (4) to be the same regardless of the local distance between $X_{i}$ and $x$. Thus, the local estimators $\beta(x)$ in (4) converge to the global estimator $\gamma$ in (2) as the bandwidths become larger. It shows that the local logit model encompasses the global parametric QMLE-RFRV.

Moreover, when the dimension $p$ of continuous variables is large $(p \geq 4)$, in general, the nonparametric estimation method has curse of dimensionality problem. As mentioned in Frölich (2006), the variance of the error term is bounded in the model with fractional response variable, and therefore, the curse of dimensionality problem does not arise in the local logit model we study in this paper. However, in the estimation of local logit model with binary response variable, Frölich (2006) assigns one common bandwidth for all discrete and another common bandwidth for all continuous regressors, and the continuous variables are scaled to the same mean and standard deviation. In our study, we apply different bandwidths for the continuous variables and only one bandwidth for all discrete variables.

\section{Simulation study}

In this section, we conduct an extensive simulation study in order to assess the finite sample properties of the proposed local logit model estimators and their robustness to various nonlinear functional forms for the conditional mean and to various symmetric and asymmetric error distributions. For the comparison purpose, we consider the QMLERFRV as the benchmark model with correct linear and nonlinear functional forms. On the other hand, there is no assumption on the conditional mean specification of local logit model. Additionally, the shape of the error distribution is assumed to be unknown for both models. We also ensure that the response variable generated is bounded in $[0,1]$ with high intensity at the boundaries zero and one, which reflect the typical features of the RR data to be modelled in the empirical application - one of the main objectives of this paper. We generate the data for two sample sizes, $n=200$ and $n=500$. 


\subsection{Experimental design}

In this experimental design, we generate seven sets of univariate and multivariate $X$ variables with different degrees of nonlinearity in the conditional mean specifications. Furthermore, the data generating processes include various distributional assumptions.

\section{A1 Univariate data generating process}

We generate the data as follows: $X_{1} \sim N(1,1), U \sim N(0,1)$, and the response variable with two-sided censoring as $Y=\max \left(1, \min \left(Y^{*}\right)\right) . Y^{*}=f\left(X_{1}\right)$ is the conditional mean specification with three different functional forms:

(U1) $Y^{*}=0.5 X_{1}+U$

(U2) $Y^{*}=X_{1}^{2}+U$

(U3) $Y^{*}=\sin \left(X_{1}\right)+U$

In other words, the univariate functional forms include linear, quadratic and sine functions.

\section{A2 Bivariate data generating process}

Generate bivariate data $\left(X_{1}, X_{2}\right)$. Then, similar to $\mathbf{A} 1, Y=\max \left(1, \min \left(Y^{*}\right)\right)$, where $Y^{*}=f\left(X_{1}, X_{2}\right)$. For a given data generating process $\mathrm{U}$ where $U \sim N(0,1),\left(X_{1}, X_{2}\right)$ and $Y^{*}$ are generated as follows:

(B1) $X_{1} \sim N(0,1), X_{2} \sim N(0,1)$, and $Y^{*}=0.2 X_{1}+0.5 X_{2}+U$

(B2) $X_{1} \sim N(0,1), X_{2} \sim N(0,1)$, and $Y^{*}=0.2 X_{1}+0.5 X_{2}^{2}+U$

(B3) $X_{1} \sim \chi_{(3)}^{2}, X_{2} \sim N(0,1)$, and $Y^{*}=0.5 \sin \left(X_{1}\right)+0.5 X_{2}+0.2 X_{2}^{2}+U$.

\section{A3 Multivariate data generating process}

We generate a multiple data set which is a mixture of continuous and discrete independent variables, which is common in many practical applications arising in economics, finance and other disciplines. 
(M1) $Y=\Phi\left(-0.02 X_{1}+\sin \left(X_{2}\right)+D_{1}+0.5 D_{2}+D_{3}+0.5 X_{1} D_{2}+U\right)$,

where $\Phi(\cdot)$ is a probit link function, $X_{1} \sim \chi_{(3)}^{2}, X_{2} \sim N(1,1), D_{1} \sim \operatorname{Ber}(1,0.75), D_{2} \sim$ $\operatorname{Ber}(1,0.4), D_{3} \sim \operatorname{Ber}(1,0.2)$, and $U$ is generated from a equally weighted mixture of $N(-2,1)$ and $N(2,1)$. Given the complexity of the functional form ${ }^{8}$, we consider only $\mathrm{n}$ $=500$.

\subsection{Simulation results}

We assess the finite sample properties of the proposed local logit model in comparison with the parametric QMLE-RFRV - the benchmark model in terms of the in-sample and out-of-sample predictabilities and the interpretability of the model estimates. We do these in the following four steps: (i) partition the full sample into in-sample and out-ofsample data; (ii) evaluate the predictability of the models using MSE and MAE criteria; (iii) repeat the above steps (i) and (ii) 100 times, then compute the average MSE and MAE; and (iv) compare the local logit model estimators with those of the benchmark model with correct model specifications. We assess these properties for the three data generating processes given in $\mathrm{A} 1$ to $\mathrm{A} 3$, and $\mathrm{n}=200$ and $\mathrm{n}=500$.

\subsubsection{Predictive performance}

Tables 1 and 2 report in- and out-of-sample predictive measures MSE and MAE of the local logit and the benchmark model for $\mathrm{n}=200$ and 500, respectively. The results show that the proposed model consistently outperforms the benchmark model in the in-sample prediction, while the out-of-sample performance of the local logit model is comparable to the benchmark model with correctly specified functional form.

The noteworthy result is that the selected bandwidths are substantially large when the true conditional mean is linear as in U1 and B1, which indicates that the local logit estimates identify the model specification correctly. In the empirical study of recovery rate modelling, we will exploit such information from the local logit estimation to "calibrate" the QMLE-RFRV model; see Section 6 for details.

Insert [Tables 1 and 2 ] here

\footnotetext{
${ }^{8}$ As there are three dummy variables, we consider only the moderate sample size to avoid the possibility of causing discontinuity in the conditional mean
} 


\subsubsection{Local logit analysis}

In this section, we study how close the local logit estimators are to those of the benchmark model with correct functional form, when the data generating process is multivariate (M1) - a mixture of continuous and discrete variables.

Let us denote the estimate of the benchmark model as:

$$
\hat{y}=\Lambda\left(\hat{\gamma}+\hat{\gamma}_{1} x_{1}+\hat{\gamma}_{2} \sin \left(x_{2}\right)+\hat{\gamma}_{3} d_{1}+\hat{\gamma}_{4} d_{2}+\hat{\gamma}_{5} d_{3}+\hat{\gamma}_{6} x_{1} d_{2}\right)
$$

and the estimate of the local logit regression is:

$$
\hat{y}=\Lambda\left(\hat{\beta}_{0}(x)+\hat{\beta}_{1}(x) x_{1}+\hat{\beta}_{2}(x) x_{2}+\hat{\beta}_{3}(x) d_{1}+\hat{\beta}_{4}(x) d_{2}+\hat{\beta}_{5}(x) d_{3}\right)
$$

First, we analyze the interaction of $x_{1}$ and $d_{2}$. That is, the effect of $x_{1}$ on y depends on $d_{2}$. We expect that the local estimate $\hat{\beta}_{1}(x)$ conditional on $d_{2}$ to be the same as the benchmark model estimate $\hat{\gamma}_{1}+\hat{\gamma}_{6}$. The plot of local estimate $\hat{\beta}_{1}(x)$ given $d_{2}=0$ appears in Figure 1, which is clearly comparable to $\hat{\gamma}_{1}$ in Figure 1c. The results suggest that both models generate more or less the same conditional marginal effect estimates of $x_{1}$. On the other hand, the local estimate $\hat{\beta}_{1}(x)$ given $d_{2}=1$ is shown in Figure $1 \mathrm{~b}$, which we compare with $\hat{\gamma}_{1}+\hat{\gamma}_{6}$ in Figure 1d. We find that the average estimates over 100 iterations in both models are approximately 0.5. These findings indicate that on average the local logit estimate captures the interaction effect between $x_{1}$ and $d_{2}$ adequately, although its variation is higher than that of benchmark model estimate.

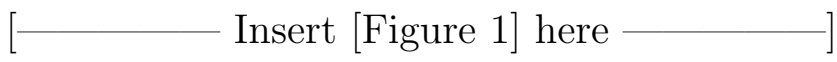

Second, consider the nonlinear component $\sin \left(x_{2}\right)$. The local marginal effect estimate $\hat{\beta}_{2}(x)$ in Figure $2 \mathrm{a}$ is compared with $\hat{\gamma}_{2} \cos \left(x_{2}\right)$ in Figure $2 \mathrm{~b}$. These figures show that the local estimate approximates some nonlinear behavior of the benchmark model. The local logit estimate shows a positive effect with diminishing rate when $x_{2}>0$ and, then, the effect is negative, which is similar to the estimate of the correctly specified benchmark model.

Third, the marginal effect estimates of discrete variables $d_{1}$ and $d_{3}$ are plotted in Figures $3 \mathrm{a}$ and $3 \mathrm{~b}$ for the local logit and the parametric QMLE-RFRV respectively. It is approximately 0.6 for both local logit and benchmark models. However, the local 
estimates have slightly higher variations than those of the QMLE-RFRV model.

[—- Insert [Figures 2, 3] here — ]

Fourth, Figure 4 shows the estimate of the interaction of $d_{2}$ and $x_{1}$. Given the correct specification of the benchmark model in (9), the marginal effect of $d_{2}$ is $\hat{\gamma}_{4}+\hat{\gamma}_{6} x_{1}$ which is shown in Figure 4b. Clearly, the effect of $d_{2}$ on the response variable is a linear function of $x_{1}$. The local logit estimate $\hat{\beta}_{4}(x)$ indicates a positive relationship between $d_{2}$ and $x_{1}$ as shown in Figure 4a, which is approximately linear.

[—- Insert [Figure 4] here - $]$

The overall results of the simulation study show that the local logit estimators can uncover the nonlinear relationship between the response variable and covariates, including various forms of nonlinearity and interactions among continuous and discrete variables.

\subsection{Robustness of the local logit model}

In this section, we evaluate the robustness of the proposed model under various assumptions for the error distribution, including bimodality and asymmetry. We consider two model specifications which include (M1) defined in Section 3.1, and (M2) defined as:

$$
Y=\Phi\left(-1.5 \sqrt{X_{1}}+\sin \left(X_{2}\right)+D_{1}+0.5 D_{2}+0.5 X_{3} D_{2}+0.2 X_{3}+d_{3}+U\right)
$$

where $X_{1} \sim \chi_{(3)}^{2}, X_{2} \sim \chi_{(1)}^{2}, X_{3} \sim N(0,2), D_{1} \sim \operatorname{Ber}(1,0.75), D_{2} \sim \operatorname{Ber}(1,0.4)$, and $D_{3} \sim \operatorname{Ber}(1,0.2)$. We consider two assumptions for the error distribution: an asymmetric $U^{(1)} \sim \chi_{(1)}^{2}$, and a bimodal $U^{(2)}$ which is generated as the equally weighted mixture of $N(-2,1)$ and $N(2,1)$.

This study estimates the MSE and MAE measures of local logit model and the benchmark model relative to those of the correctly specified parametric QMLE-RFRV model for the purpose of performance assessment. Note that the QMLE-RFRV with a standard linear functional form - benchmark model used here for the comparison purpose. Specifically, if the relative MSE and MAE are equal to or less than one, then the model performance is the same or better than the correctly specified QMLE-RFRV. We set three sample sizes, $n=200,500$ and 1,000, where the evaluations are made in the both in-sample and out-of-sample data. 
The in-sample performance measures - the relative MSE and MAE - of the proposed local logit model are reported in Panel (a) of Tables 3 and 4 respectively. These relative measures are consistently lower than those of the parametric regression. The results also show that both relative MSE and MAE are mostly less than or equal to 1.00 for both asymmetric and bimodal error distributions. On the other hand, the QMLE-RFRV benchmark model - performs poorly for asymmetric error distribution, with the both MSE and MAE being greater than 1.00 and close to 2.00 in many cases.

Panel (b) of Tables 3 and 4 reports the out-of-sample performance measures of the models. The local logit model continues to outperform the parametric regression in most cases. Additionally, the local logit tends to have substantially lower MSE and MAE for the Chi-squared error assumption compared with the bimodal error distribution. Moreover, we notice that the local logit model has relatively large MSE and MAE for bimodal distribution for a small sample size $\mathrm{n}=200$, while vast improvements are observed for the larger sample sizes.

[- Insert [Tables 3 and 4$]$ here -$]$

\section{Specification testing}

In this section, we briefly discuss a specification test for the null hypothesis that the parametric QMLE-RFRV model with a given specification fits the RR data well against the alternative hypothesis that the local logit model fits the data well. The testing procedure employs the generalized maximum likelihood ratio (Fan, Zhang, \& Zhang, 2001) and is augmented with a bootstrap method for calculating the p-value of the test statistic. The test statistic is defined as:

$$
T S=\frac{R S S_{0}-R S S_{1}}{R S S_{1}}
$$

where $R S S_{0}$ is the residual sum square under the null hypothesis which is $\frac{\sum_{i=1}^{n}\left(Y_{i}-\Lambda\left(X_{i}^{\prime} \hat{\gamma}\right)\right)^{2}}{n}$; and $R S S_{1}$ is under the alternative which is $\frac{\sum_{i=1}^{n}\left(Y_{i}-\Lambda\left(X_{i}^{\prime} \hat{\beta}(x)\right)\right)^{2}}{n}$. The null hypothesis is rejected if the p-value of the TS is less than the nominal level. To compute the p-value, we apply the wild bootstrap procedure as follows:

1. Under the null hypothesis, generate $Y_{i}^{*}=\Lambda\left(X_{i}^{\prime} \hat{\gamma}+e_{i}^{*}\right)$ for each $i=1, \ldots, n$, where 
$e_{i}^{*}$ is generated as follows:

- Estimate the residual $\hat{e}_{i}=\Lambda^{-1}\left(Y_{i}^{(\nu)}\right)-X_{i} \hat{\gamma}$ where $Y_{i}^{(\nu)}=\frac{Y_{i}+\nu}{1+2 \nu}$, and $\nu$ is a small arbitrary value ${ }^{9}$.

- Obtain $e_{i}^{*}=\left(\hat{e}_{i}-\frac{1}{n} \sum_{i=1}^{n} \hat{e}_{i}\right) \cdot \eta_{i}$ where $\left\{\eta_{i}\right\}$ is a sequence of independent and identically distributed random variables drawn from $\mathrm{N}(0,1)$.

2. Use the dataset $\left\{\left(Y_{i}^{*}, X_{i}\right): i=1, \ldots, n\right\}$ to estimate the models under both null and alternative hypotheses. Then, the test statistic is calculated as $T S^{*}=\frac{R S S_{0}^{*}-R S S_{1}^{*}}{R S S_{1}^{*}}$.

3. Repeat Steps 1 and $2 \mathrm{~B}$ times to draw the empirical distribution for $T S^{*}$. Then, the p-value is computed by $\frac{1}{B} \sum_{b=1}^{B} I\left(T S_{b}^{*} \geq T S\right)$, where $I(\cdot)$ is an indicator function and $T S_{b}^{*}$ is calculated based on the b-th bootstrap sample.

\section{Data and the preliminary analysis}

In this section, we summarize the empirical recovery rate data as well as a summary statistics and a preliminary data analysis. These indicate some stylized facts and typical features of the RR data and the covariates.

The dataset on realized recovery rate is obtained from the Moody's Ultimate Recovery Database, which have been used in several studies, such as Qi and Zhao (2011), Altman and Kalotay (2014) and Siao, Hwang, and Chu (2015), among others. The data has 3,573 cross-sectional recovery rates from the US corporate loans that had been defaulted from 1994 to 2012 . The data shows that $40 \%$ of the loans is full recovery, followed by $5 \%$ of the complete loss. This forms the bimodal property in the density due to the high masses at both boundaries zero and one, as shown in Figure 5.

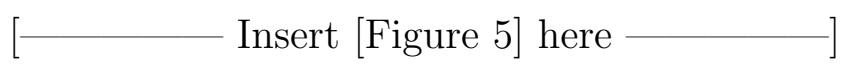

Moody's also provides the debt characteristics prior to default including debt cushion $^{10}$, the instrumental rank in capital structure, types of commercial loans, subordination degree of bonds, and collateral status. We obtain the St. Louis Fed Financial Stress In-

\footnotetext{
${ }^{9}$ This allows $\Lambda^{-1}\left(Y_{i}^{(\nu)}\right)$ to be possible.

${ }^{10}$ Moody's defines debt cushion as the ratio of the face value of a claim to the total debt below it. The high DC reflects the low outstanding debt in the company capital structure.
} 
$\operatorname{dex}(\mathrm{SI})$ from the US federal reserve bank of St. Louis ${ }^{11}$. This index measures the stress in the US financial market and economy, which is constructed by using 17 different key indices, such as federal funds rate, corporate credit risk spread, interest rate and inflation (Kliesen, Smith, et al., 2010). The average value of index is set at zero in the late 1993, and a positive SI indicates the above-average financial market or economic stress condition. For defaulted date of each loan, the stress index is matched with the date to reveal the economic or financial market condition at the time of default. We observed that SI is mostly between -1 and 1 , although a few SI is above 1.2 reflecting extreme stressful economy which only observed in the recent financial crisis (GFC). Figure 6 illustrates the movement of the annual averages SI in the last decades, which reflects several economic conditions such as Dot-Com crisis (1999 - 2003), economic expansion (2004-2006), as well as the global financial crisis (2007-2010). The figure also shows the negative relationships between SI and the recovery rate.

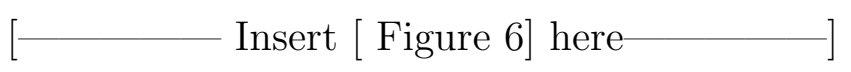

Table 5 provides the summary statistics of each variable as well as the contingency table of the covariates and the recovery rate. There are five determinants, of which three categorical variables, types of loan, instrumental rank and collateral status (see Panel A in Table 5), and two continuous variables DC and SI (see Panel B). In the first row, the reported figures were the recovery rates at the $0.05,0.25,0.5,0.75$, and 0.95 quantiles. The other rows indicate the frequency distributions of recovery rate conditional on each category of categorical variables, followed by those conditional on discretised values of the two continuous variables.

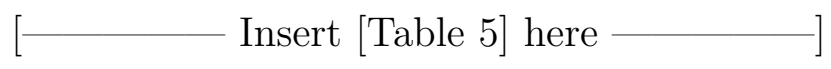

Panel A(i), our data has five different types of the defaulted loans, where the data has $42 \%$ of commercial loans ${ }^{12}$ and $58 \%$ of bonds ${ }^{13}$. Considering the average RR of each type, the revolving loan has the highest rate, while the junior and subordinate bonds are the most risky types of loan with the lowest average of 0.24 . We also find that the recovery rates of the commercial loans and the senior secured bond tend to have negative skewness

\footnotetext{
${ }^{11}$ Federal Reserve Bank of St. Louis, St. Louis Fed Financial Stress Index@ [STLFSI], retrieved from FRED, Federal Reserve Bank of St. Louis; https://fred.stlouisfed.org/series/STLFSI

${ }^{12}$ which are term loan and revolving loan

${ }^{13}$ There are four types of the bonds defined by their seniority
} 
compared to the remaining loans, due to the relatively high medians and high masses at the upper quantiles.

The instrumental rank generally indicates the repayment priority in the capital structure $^{14}$. Therefore, we find that the averages of the recovery rate decrease as Rank increases, in Table 5, Panel A(ii). Also, as most commercial loans generally have Rank 1, the contingency table shows that the RR densities of Rank 1 is similar to those of the commercial loans.

Lastly, collateral is the main source of fund to repay the outstanding defaulted debt. Panel A(iii) shows that the collateralised loan has substantial higher average recovery rate than the uncollateralised loan. $50 \%$ of the uncollateralised loan can recover less than $20 \%$ of the total loss, while more than a half of collateralised loans can recover more than $90 \%$.

Panel B represents the preliminary analysis of the relationships the continuous variables and RR. First, we partition the defaulted loan based on the level of DC. Debt Cushion, as suggested by Van de Castle and Keisman (1999), is a facility-level metric that captures not only the rank of debt in capital structure, but the degree of its subordination as a proportion of total claims. This, in turn, reflects the liquidity available for a liquidation. Table 5 shows that $46 \%$ of the data has zero DC, where the average RR of them is 0.4 . If the $\mathrm{DC}$ is greater than 0.5 , the average $\mathrm{RR}$ can be as high as 0.8 , also more than three fourth of them has almost full recovery on average, compared to 0.07 for the loan with zero DC.

Lastly, the recovery rate is partitioned by the given levels of SI in Panel B(ii). We denote the range of DC as negative SI, $0<S I<1$, and $S I \geq 1$, which represent low, high, and substantial high stressed periods, respectively. Based on the average RR, the recovery rate during the low stress is the lowest at 0.7 , while the rates are similar at approximately 0.5 for high and substantial high stress periods. During the good economic condition, more than a half of the defaulted loan can be recovered more than $80 \%$ of the total loss compared to $40 \%$ for the otherwise periods. We, then, expect the negative effect of SI on RR. It can be also notice that the densities of RR during high and extremely high economic stress are distributed as similar as one another.

\footnotetext{
${ }^{14}$ To recover the defaulted loss after declared bankruptcy of the borrowers, their assets will be liquidated and then allocated to repay the lenders, which is prioritised by the instrumental rank.
} 


\section{Empirical results}

The local logit model is applied to RR dataset to uncover the nature of the underlying unknown nonlinear RR-covariates relationship, conduct marginal and interaction effects analysis, and generate RR predictions. The results of this empirical investigation will be utilized to improve the specification of the parametric QMLE-RFRV model - calibrated model.

\subsection{Bandwidth selection}

We estimate the local logit model for the full dataset of 3,573 defaulted loans. The local logit regression for the $\mathrm{RR}$ data is specified as:

$$
\begin{aligned}
y=E(Y \mid X=x)= & \Lambda\left(x^{\prime} \beta(x)\right) \\
= & \Lambda\left(\beta_{0}(x)+\beta_{D C}(x) \cdot D C+\beta_{S I}(x) \cdot S I\right. \\
& +\sum_{d=2}^{6} \beta_{\text {Type }}(d)(x) \cdot \text { Type }^{(d)}+\sum_{d=2}^{4} \beta_{\text {Rank }^{(d)}}(x) \cdot \operatorname{Rank}^{(d)} \\
& \left.+\beta_{C o l}(x) \cdot C o l\right)
\end{aligned}
$$

where $\beta(x)=\left(\beta_{0}(x), \beta_{D C}(x), \ldots, \beta_{C o l}(x)\right)^{\prime}$ is a vector of unknown parameters, which are functions of the entire set of covariates $x$. Type $e^{(d)}$ and $\operatorname{Rank}^{(d)}$ are dummy variables representing each category of discrete variables Type and Rank, respectively; See Table 5 for more details. For a comparison purpose, we estimate the benchmark model which is the standard linear QMLE-RFRV, denoted as $\Lambda\left(x^{\prime} \gamma\right)$.

The local logit parameters are estimated with the kernel function (5) for continuous variables DC and SI, and kernel function (6) for categorical variables Type, Rank and Col. The bandwidth is selected by the leave-one-out least-squares cross-validation method (7). Let us define,

$$
H=\left(h_{1}, h_{2}, \ell_{1}\right)
$$

where $H$ is $3 \times 1$ vector of the bandwidths, $h_{1}$ and $h_{2}$ are associated with DC and SI, respectively, and $\ell_{1}$ is a single bandwidth for all three categorical variables: Rank, Type and Col. $H=(0.11,1.27,1.00)$ is the set of selected optimum bandwidths. We estimate 
the local logit model with the selected bandwidths for the full dataset and find that MSE of the local logit model is 0.076 , whereas it is 0.089 for the benchmark model QMLERFRV. The results indicate that the local logit is a better fit for the RR data than the benchmark model.

\subsection{Local logit analysis}

The marginal effects of continuous variables and discrete variables are analysed in the local logit and the parametric QMLE-RFRV models.

(i) Local logit estimates of continuous variables

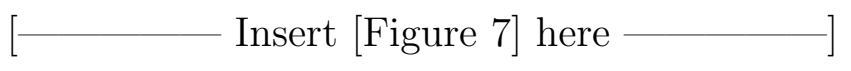

Figure 7a shows the local logit estimate of DC is a nonlinear function of DC, denoted as $\hat{\beta}_{D C}(x)$, while the QMLE-RFRV estimate $\hat{\gamma}_{D C}$ represented by the solid horizontal line. In the local logit model, the marginal effect on DC depends on the level of DC, whereas it is constant in the parametric model.

In the local logit model, the effect of $\mathrm{DC}$ on RR increases somewhat linearly for $0<D C<0.6$, reaching the highest impact when $\mathrm{DC}=0.6$, followed by a decreasing effect on the $\mathrm{RR}$ of the defaulted loans for $0.6 \leq D C<0.8$ reaching the lowest effect at $D C=0.8$. There onwards, the effect increases for $\mathrm{DC}>0.8$. These results imply that the defaulted loans with $0<D C<0.6$ tend to be more effectively responsive to an increase in additional DC than the loan with higher DC. In comparison to the parametric estimate of 2.5, the effect of DC on RR is mostly positive as expected and the average local logit estimate $\hat{\beta}_{D C}(x)$ is also close to 2.5 (Figure 7a).

To analyze the effect of SI on RR, $\hat{\beta}_{S I}(x)$ and $\hat{\gamma}_{S I}$ are plotted (solid line) in Figure 7b. To explain the marginal effect of SI on RR, we consider three ranges of the SI: low SI as $\mathrm{SI}<0$, high SI as $0<S I<1.5$, and the crisis $\mathrm{SI}$ as $\mathrm{SI}>1.5$, indicating good, poor and (global financial) crisis economic conditions. The effect of SI on RR is negative and increasing with SI and then it becomes positive and increasing for SI $>1.5$. The variation of the effect of SI on RR is very high for the low values of SI. This result indicates that $\mathrm{RR}$ is more sensitive to the change in the economic conditions during the low SI 
period, compared to high SI and the crisis. The variation of the local logit estimate shows that although SI has a nonlinear negative effect on RR, the magnitude of the effects are different depending on the characteristics of each loan. The relatively small variation of the local estimate observed for high SI implies that the effect of high SI is less dependent on the loan characteristics than for the low SI and the crisis SI. In practice, these findings indicate by the change in behavior and expectations of both banks and borrowers during the economic downturn $(0<S I \leq 1.5)$. Lenders would have similarly adopted more conservative financial strategies preparing to a pessimistic scenario. This leads to the smaller negative effect of high SI on $\mathrm{RR}$ with lower variation.

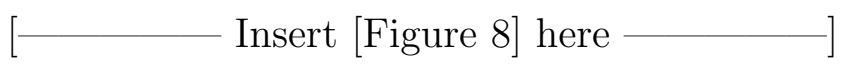

Furthermore, we consider SI $=\{-1,0,1\}$ and study the effect of DC on RR for the loans with $($ Type $=2, \operatorname{Rank}=1, \mathrm{Col}=1$ ) across various economic conditions measured by SI. The plot in Figure 8 indicates the RR is nonlinear function of DC. The marginal effect of $\mathrm{DC}$ on $\mathrm{RR}$ is zero for $\mathrm{DC}<0.3$, and positive \& increasing until $\mathrm{DC}=0.6$, and then nearly zero for DC $>0.6$. On the other hand, SI has a negative impact on RR. For example, if we consider a loan with $\mathrm{DC}=0$, then the $\mathrm{RR}$ is $0.63,0.75$, and 0.90 respectively for high stress period $(\mathrm{SI}=1)$, neutral period $(\mathrm{SI}=0)$, and low stress period $(\mathrm{SI}=-1)$ respectively. Figure 8 also shows that the negative effect of SI on the loan with low $\mathrm{DC}$ is stronger than the loan with higher $\mathrm{DC}$, as $\mathrm{RR}$ of the given loan with $\mathrm{DC}=1$ is approximately one for all levels of SI. The loan with high level of DC is not very sensitive to the change in the economic condition compared to that with the lower DC.

\section{(ii) Local estimates of discrete variables}

We turn to the analysis of the local estimates of the discrete variables including type of loan; instrumental rank; and collateral status. The estimates indicate the levels of riskiness of each category in comparison to the reference category ${ }^{15}$. Specifically, a negative estimate means the category of interest has lower RR (higher risk) than the reference category, given other variables held constant.

Table 6 compares the median of local logit estimates of all discrete variables with the coefficients estimates of QMLE-RFRV. The result shows that the median of the local estimates and the QMLE-RFRV estimates are more or less the same. These results imply

\footnotetext{
${ }^{15}$ The reference categories of Type, Rank and Col are given in Table 5
} 
that the local estimators for the discrete variables contain somewhat similar information as the parametric estimators.

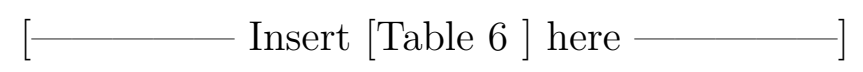

The local logit estimates of all discrete variables are presented in Figure 9 and their signs are mostly in line with expectation. However, there are some unexpected positive estimates for the local estimates of senior secured bond (Type 3), senior unsecured bond (Type 5) in Figure 9a; and unexpected negative estimates for Col in Figure 9c. In general, both types of senior bonds are expected to have lower RR than the term loan due to the priority in the credit capital structure, hence only a negative sign is expected. On the other hand, the collateralised loan is commonly expected to have a higher RR than the loan without collateral, then the positive effect is expected. As shown earlier in the simulation study, the unexpected signs of the estimates maybe due to the presence of potential interaction effects.

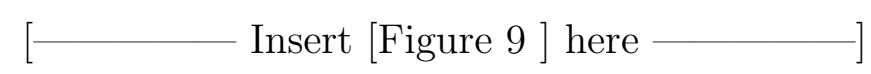

We find that there are some significant relationships between DC and both local estimates of Type $=\{3,5\}$ and $\mathrm{Col}=1$. Figure 10 shows that the effects of both senior bonds are highly dependent on the levels of DC, which indicate the interactions between DC and both senior bonds. First, for the senior secured bond, Figure 10a shows that the unexpected positive estimates for the defaulted loan with $0.2<D C<0.6$. Second, for the senior unsecured bond, the expected negative signs are observed only for the loan with $0.1<D C<0.5$ in Figure 10b.

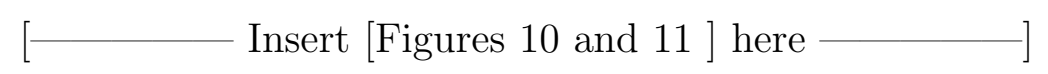

To explain the unexpected negative estimate of Col, see Figure 11 which shows the relationship between the local estimates of $\mathrm{Col}$ and the levels of DC. The clear pattern emerges in Figure 11a, the estimates are negative, when the defaulted loan has DC between 0.2 and 0.5 .

In Table 7, we report the results of the partitioning empirical RR data based on the findings of the interaction effect analysis. The result confirms that the local logit analysis can uncover the some underlying true interaction effects observed in the empirical data. For example, in Panel A, we compare the average RR of the senior secured bond with those of the collateralized term loan (reference category) for various ranges of DC . We find that, although the average RR of the term loan is mostly higher than that of senior 
secured bond, only the bond with $0.2 \leq D C<0.6$ has a higher $\mathrm{RR}$ than the term loan. This finding is consistent with the positive local logit estimate of the senior secured bond plotted in Figure 10a.

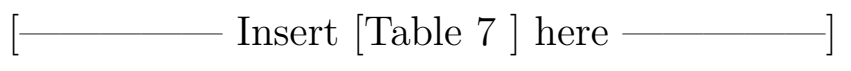

\subsection{Calibrated QMLE regression for fractional response variable}

Table 8 reports the estimates of the improved specification of the QMLE-RFRV - calibrated model. The variables and interaction terms (Column 2 of Panels A, B, C and D, Table 8) of the calibrated model were obtained from the results of local logit analysis ${ }^{16}$. In Panel B, the parameter estimates of $\gamma_{S I_{1}}, \gamma_{S I_{2}}$, and $\gamma_{S I_{3}}$ represent the effects of SI for low SI, high SI and the crisis SI, respectively. The results show that negative effects are the strongest for the low SI followed by the high SI, and the effect becomes insignificant during the crisis SI, which is consistent with our previous findings in the local logit analysis. Furthermore, the interaction effects between DC and the senior secured bond are captured by the parameters $\gamma_{T_{31}}, \gamma_{T_{32}}$, and $\gamma_{T_{33}}$ in Panel C. The results show that only $\gamma_{T_{32}}$ estimate is significantly positive, which represents the interaction effect between senior secured bond and $D C \in[0.2,0.6)$. This means that the senior secured bond with $0.2 \leq D C<0.6$ is likely to have higher RR than the term loan, which is consistent with our previous findings in local logit model. These results show that the behaviours of the parameter estimates in the calibrated model are more or less the same as those of the the local logit estimates.

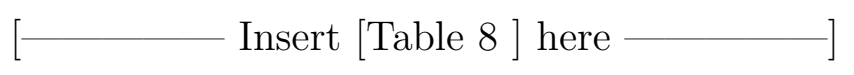

Moreover, we test the null hypothesis that the calibrated QMLE-RFRV fits the data against the alternative hypothesis that the local logit fits the data well, by applying the wild bootstrap-based specification test with 1,000 iterations. The calibrated QMLERFRV model (Table 8 ) is not rejected at the $5 \%$ nominal level, as the p-value computed by the bootstrap method 0.09. This test provides statistical evidence that the calibrated model specification fits the RR data well.

\footnotetext{
${ }^{16} \mathrm{We}$ also found the interaction term between Type $=2$ and DC.
} 


\subsection{Out-of-sample predictive performance}

In this section, we compared the out-of-sample predictability of the local logit model, the calibrated QMLE-RFRV model and the standard QMLE-RFRV model. In this study, we evaluate the point predictive and the quantile predictive performances of these three models.

\subsubsection{Predictive performance criteria}

\section{Point prediction evaluation}

We use three methods to partition the full samples into in- and out-of-samples and assess the sensitivity of the models' predictions to these methods. The three methods include:

(DF1) Partition the full sample randomly into pre-specified 70:30 ratio of in-sample:outof-sample, for 1,000 iterations. Although, this is a standard evaluation of out-ofsample prediction, the overfitting issue is not properly addressed. According to our empirical RR data, one borrower could have several defaulted loans. By randomly partition the full data, it allows the overlapping information, as the information of a borrower could be in both in- and out-of-sample data. This leads to the overfitting problem (Kalotay \& Altman, 2016).

(DF2) Partition the full sample into, for example, the in-sample period 1994-2005, and the out-of-sample period 2006-2012. This way of partitioning ensures that there is no overlapping observations in the both samples. This definition also mimics the application of RR predictive model in practice, as banks would want to use the full observed data to predict RR in the forthcoming years.

(DF3) Select any particular year as the out-of-sample period, and the remaining years as the in-sample period. For example, the out-of-sample period is the start of the GFC, 2008, then the in-sample period is 1994-2007 and 2009-2012. This way of partitioning the in- and out-of-sample is very useful to predict $R R$ at the various phases of the economic cycle.

\section{Quantile prediction evaluation}

In this method, we evaluate the predictive performance of the models at various quantiles 
of the simulated RR portfolio distribution of the out-of-sample; see Altman and Kalotay (2014) for details. The following re-sampling procedure is employed to construct the RR portfolio distribution:

(i) Define the in-sample data period 1994-2004 and the out-of-sample period 2004-2012

(ii) Draw a random sample of $100 \mathrm{RRs}$ from the out-of-sample data with replacement. Assign each loan a $\$ 1.00$ face value and construct an equally-weighted portfolio of the selected RRs. This RR portfolio represents the money that is recovered from $\$ 100.00$ portfolio's face value.

(iii) Predict the selected out-of-sample RR by the benchmark QMLE-RFRV model, local logit model, and calibrated QMLE-RFRV model. Then, the predicted RR portfolio is constructed for each model

(iv) Repeat (ii) and (iii) above 10,000 times and construct a simulated RR portfolio distributions for the three models under investigation

The model performance is evaluated by the predictive error of the simulated RR portfolio at various quantiles of the distribution.

\subsubsection{Comparison of predictive performances}

\section{Point prediction accuracy}

We adopt the data partitioning method DF1, the out-of-sample MSE and MAE of the local logit model are 0.0824 and 0.2750 , respectively. For the calibrated linear model, they are 0.0854 and 0.2880 , receptively. On the other hand, the benchmark model has the highest predictive errors, 0.0964 and 0.3246 . These results indicate that the local logit model outperforms others.

The results of the out-of-sample evaluation of the models for DF2 are reported in Table 9, which include the predictive performances of 11 different out-of-sample windows from 2001 to 2012. For the first window, we estimate the models for the in-sample period 1994 to 2000, and evaluate the predictions of out-of-sample period 2001 to 2012. Then, the in-sample window is continually expanded by each calendar year until the eleventh window in-sample period is 1994 to 2010 and the out-of-sample period is only 2011-2012. The MSE and MAE of the predictions for each window are reported in Table 9. 


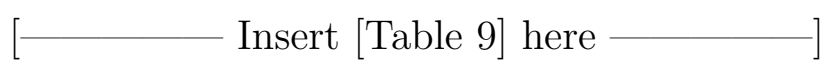

The result shows that the proposed local logit model has the highest predictive accuracy, followed by the calibrated model. The benchmark model outperforms the proposed model only in the two out-of-sample windows of 2002-2012 and 2010-2012 under the both MSE and MAE criteria at the $5 \%$ level of significance (Table 9). This table also provides the average and variance of MSE and MAE over 11 windows. The MSE and MAE averages of the proposed local logit model as well as their variances are consistently lower than those of the benchmark model.

Noticeably, the differences in MSE among three models are large for the out-of-sample predictions between 2004 and 2008 in Table 9. These years are crucial, since they partially cover the global financial crisis period 2007 to 2010. The benchmark model is highly sensitive to the crisis year compared to the non-parametric and the calibrated models. The MSE and MAE are very large during the crisis period for the benchmark model. The low accuracy of the benchmark model during the GFC could be due to the unexpected shock with substantially high level of SI. As a linear model, the constant negative effect of SI could lead to the underprediction of RR during the crisis.

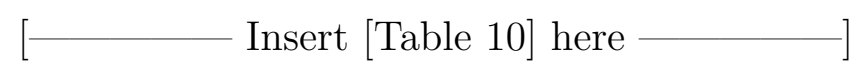

The results of the point prediction evaluations of the three models for DF3 are presented in Table 10, where we predict RR every year from 2000 to 2011. Table 10 shows that the local logit regression consistently outperforms the benchmark regression. The MSE and MAE averages of the proposed model across 11 years are 0.087 and 0.224 , compared to 0.096 and 0.235 for the benchmark model. The benchmark model prediction outperforms the proposed model only in 2010 and 2011. The calibrated model mostly outperforms the benchmark model and its performance is comparable to that of local logit model. As far as the economic cycle is concerned, the local logit model and the calibrated QMLE-RFRV model have comparable performance and outperform the benchmark model at all window sizes, and the MSEs of those former models are substantially lower than the benchmark model during the GFC period. On the other hand, we observe that the benchmark model yields relatively high MSE during the recent GFC periods (2007-2009) when SI level is at its peak. 


\section{Quantile prediction accuracy}

We evaluate the performances of the models at various quantiles of the simulated portfolio distribution. The results in Table 11 compare RRs at the $0.05,0.25,0.5,0.75$, and 0.95 quantiles of the observed RR portfolio distribution with those of the predicted portfolio distributions. The local logit model and the calibrated model predict RR portfolio at the five selected quantiles of the distribution more precisely than the benchmark model. For example, at the 0.5 quantile of the portfolio distribution, the actual portfolio can recover $\$ 63.96$ from $\$ 100.00$ face value, while the predictions by the both proposed model and the calibrated model are approximately $\$ 61.30$ compared to the benchmark model prediction of $\$ 67.71$. This implies that the benchmark model is more likely to overestimate the RR portfolio value compared to other two models. Also we find that the local logit outperforms the other models for the high risk portfolios (at the low quantiles) followed by the calibrated model.

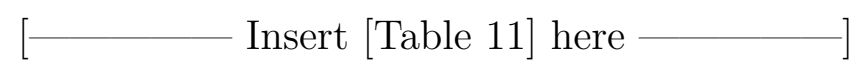

In summary, the proposed local logit model outperforms the other two models as indicated by all predictive performance measure criteria by the both point and quantile predictions. We also find that the calibrated model has slightly lower predictability than the proposed model, and outperforms the benchmark model.

\section{Conclusion}

In this study, we propose a nonparametric local logit model for $[0,1]$ bounded response variable, assess their finite sample properties relative to the QMLE regression for fractional response variable (QMLE-RFRV) - the benchmark model. These two models are then applied to empirical RR data and covariates. The results of the marginal and interaction effect analyses of the local logit model are utilised to calibrate the QMLE-RFRV model. The in-sample and out-of sample predictive performances of the three models are assessed using MSE and MAE measures. The main findings of this study are the following:

First, an extensive simulation study establishes that the properties of local logit model estimates are as good as than the correctly specified parametric model in moderate sample sizes and they are robustness to asymmetric and bimodal error distributions. Second, we apply local logit regression to model RR data, which uncovers the underlying nonlin- 
ear RR data and covariates relationship including interaction effects among covariates. Third, we exploit the results of local logit model to improve the parametric QMLE-RFRV model specification, which we call calibrated model. The calibrated model is nonlinear in variables which includes some useful interaction terms. Fourth, we assess the in-sample and the out-of-sample RR predictability of the local logit model and the calibrated model in comparison to the standard parametric model. The results show that the local logit model outperforms the others. In addition, the calibrated model is comparable to local logit model in the predictive performance. An attractive feature of the local logit and calibrated models is that they outperform the benchmark model in the out-of-sample RR prediction during the crisis period. Our findings are useful to applied researchers and practitioners who are unfamiliar with the nonparametric machinery, and banks to design treatment program for their borrowers.

\section{Appendix A: Simulations results}

\section{Tables and figures}

\begin{tabular}{|c|c|c|c|c|c|c|c|c|}
\hline \multirow{3}{*}{$\begin{array}{c}\mathrm{n}=200 \\
\text { Specification }\end{array}$} & \multicolumn{4}{|c|}{ In-sample } & \multicolumn{4}{|c|}{ Out-of-sample } \\
\hline & \multicolumn{2}{|c|}{ Benchmark } & \multicolumn{2}{|c|}{ LL } & \multicolumn{2}{|c|}{ Benchmark } & \multicolumn{2}{|c|}{ LL } \\
\hline & MSE & MAE & MSE & MAE & MSE & MAE & MSE & MAE \\
\hline U1 & 0.0156 & 0.0720 & 0.0156 & 0.0720 & 0.0161 & 0.0722 & 0.0161 & 0.0722 \\
\hline U2 & 0.0195 & 0.0825 & 0.0182 & 0.0811 & 0.0199 & 0.0825 & 0.0200 & 0.0831 \\
\hline U3 & 0.0325 & 0.1362 & 0.0312 & 0.1361 & 0.0331 & 0.1382 & 0.0333 & 0.1399 \\
\hline B1 & 0.0211 & 0.1022 & 0.0211 & 0.1022 & 0.0215 & 0.1028 & 0.0215 & 0.1028 \\
\hline B2 & 0.0312 & 0.1299 & 0.0298 & 0.1299 & 0.0314 & 0.1300 & 0.0322 & 0.1312 \\
\hline B3 & 0.0260 & 0.1109 & 0.0234 & 0.1102 & 0.0256 & 0.1129 & 0.035 & 0.1271 \\
\hline
\end{tabular}

Table 1: In-sample and out-of-sample predictions of models in the simulation study with small sample size Note: The benchmark model is the standard QMLE-RFRV. The local logit regression is denoted as LL 


\begin{tabular}{|c|c|c|c|c|c|c|c|c|}
\hline \multirow{3}{*}{$\begin{array}{c}\mathrm{n}=500 \\
\text { Specification }\end{array}$} & \multicolumn{4}{|c|}{ In-sample } & \multicolumn{4}{|c|}{ Out-of-sample } \\
\hline & \multicolumn{2}{|c|}{ Benchmark } & \multicolumn{2}{|c|}{ LL } & \multicolumn{2}{|c|}{ Benchmark } & \multicolumn{2}{|c|}{ LL } \\
\hline & MSE & MAE & MSE & MAE & MSE & MAE & MSE & MAE \\
\hline U1 & 0.0121 & 0.0682 & 0.0121 & 0.0682 & 0.0112 & 0.0691 & 0.0112 & 0.0691 \\
\hline U2 & 0.0191 & 0.0841 & 0.0187 & 0.0827 & 0.0181 & 0.0811 & 0.0182 & 0.0811 \\
\hline U3 & 0.0333 & 0.1355 & 0.0326 & 0.135 & 0.0325 & 0.1386 & 0.0326 & 0.1388 \\
\hline B1 & 0.2041 & 0.0958 & 0.2041 & 0.0958 & 0.0207 & 0.1005 & 0.0207 & 0.1005 \\
\hline B2 & 0.0309 & 0.1255 & 0.0301 & 0.1256 & 0.0313 & 0.1291 & 0.0314 & 0.1300 \\
\hline B3 & 0.0261 & 0.11 & 0.0241 & 0.1021 & 0.0252 & 0.1123 & 0.0259 & 0.1151 \\
\hline M1 & 0.1100 & 0.2731 & 0.109 & 0.2722 & 0.1154 & 0.2781 & 0.1162 & 0.2812 \\
\hline
\end{tabular}

Table 2: In-sample and out-of-sample predictions of models in the simulation study with moderate sample size

Note: The benchmark model is the standard QMLE-RFRV. The local logit regression is denoted as LL

\begin{tabular}{|c|c|c|c|c|c|c|}
\hline \multirow[b]{2}{*}{ Error $(\mathrm{U})$ distribution } & \multicolumn{2}{|c|}{$\mathrm{n}=200$} & \multicolumn{2}{|c|}{$\mathrm{n}=500$} & \multicolumn{2}{|c|}{$\mathrm{n}=1000$} \\
\hline & Benchmark & LL & Benchmark & LL & Benchmark & LL \\
\hline \multicolumn{7}{|c|}{ Panel (a): In-sample prediction } \\
\hline \multicolumn{7}{|l|}{ Specification: M1 } \\
\hline Chi-square & 1.9618 & 0.7603 & 1.8828 & 0.9218 & 1.8645 & 0.9773 \\
\hline Bimodal & 1.0893 & 1.1003 & 1.0844 & 0.9592 & 1.0839 & 0.9838 \\
\hline \multicolumn{7}{|l|}{ Specification: M2 } \\
\hline Chi-square & 1.3811 & 0.9206 & 1.3685 & 0.8512 & 1.1466 & 0.9857 \\
\hline Bimodal & 1.0615 & 1.0392 & 1.0600 & 1.0296 & 1.0569 & 0.9350 \\
\hline \multicolumn{7}{|c|}{ Panel (b): Out-of-sample prediction } \\
\hline \multicolumn{7}{|l|}{ Specification: M1 } \\
\hline Chi-square & 1.9178 & 1.4636 & 1.9816 & 1.2444 & 1.9005 & 1.1072 \\
\hline Bimodal & 1.0854 & 1.1191 & 1.0800 & 1.0501 & 1.0750 & 1.0304 \\
\hline \multicolumn{7}{|l|}{ Specification: M2 } \\
\hline Chi-square & 1.3727 & 1.4701 & 1.3553 & 1.2094 & 1.3713 & 1.1466 \\
\hline Bimodal & 1.0491 & 1.0835 & 1.0572 & 1.0503 & 1.0527 & 1.0464 \\
\hline
\end{tabular}

Table 3: The mean square error of the local logit model relative to correctly specified QMLE-RFRV model

Note: The benchmark model is the standard linear QMLE-RFRV model. LL is the proposed local logit model. The relative MSE is the MSE of the given model relative to the correctly specified QMLE-RFRV model. The error $U^{(1)} \sim \chi_{(1)}^{2}$ asymmetric distribution. The error $U^{(2)}$ generated from the equally weighted mixture of $N(-2,1)$ and $N(2,1)$ - bimodal distribution. 


\begin{tabular}{lcccccc}
\hline \multicolumn{2}{c}{$\mathrm{n}=200$} & \multicolumn{2}{c}{$\mathrm{n}=500$} & \multicolumn{2}{c}{$\mathrm{n}=1000$} \\
\hline Error (U) distribution & Benchmark & LL & Benchmark & LL & Benchmark & LL \\
\hline $\begin{array}{l}\text { Panel (a): In-sample prediction } \\
\text { Specification: M1 }\end{array}$ & & & & & \\
Chi-square & 1.5536 & 0.8819 & 1.5366 & 0.9959 & 1.5341 & 1.0272 \\
Bimodal & 1.0664 & 1.0524 & 1.0622 & 0.9962 & 1.0627 & 1.0131 \\
Specification: M2 & & & & & & \\
Chi-square & 1.2464 & 0.9099 & 1.2410 & 0.9089 & 1.2370 & 1.0050 \\
Bimodal & 1.0408 & 1.0148 & 1.0413 & 1.0250 & 1.0380 & 0.9618 \\
\hline $\begin{array}{l}\text { Panel (b): Out-of-sample prediction } \\
\text { Specification: M1 }\end{array}$ & & & & & \\
Chi-square & 1.5434 & 1.1824 & 1.5612 & 1.1310 & 1.5219 & 1.0807 \\
Bimodal & 1.0627 & 1.0597 & 1.0594 & 1.0412 & 1.0563 & 1.0357 \\
Specification: M2 & & & & & & \\
Chi-square & 1.2376 & 1.1679 & 1.2301 & 1.0880 & 1.2440 & 1.0859 \\
Bimodal & 1.0364 & 1.0404 & 1.0397 & 1.0356 & 1.0360 & 1.0219 \\
\hline
\end{tabular}

Table 4: The mean absolute error of the local logit model relative to correctly specified QMLE-RFRV model

Note: The benchmark model is the standard linear QMLE-RFRV model. LL is the proposed local logit model. The relative MAE is the MAE of the given model relative to the correctly specified QMLE-RFRV model. The error $U^{(1)} \sim \chi_{(1)}^{2}$ asymmetric distribution. The error $U^{(2)}$ generated from the equally weighted mixture of $N(-2,1)$ and $N(2,1)$ - bimodal distribution.

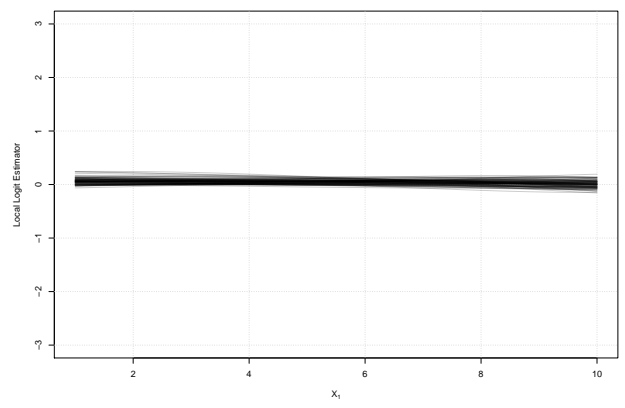

(a) Local logit regression, D2 $=0$

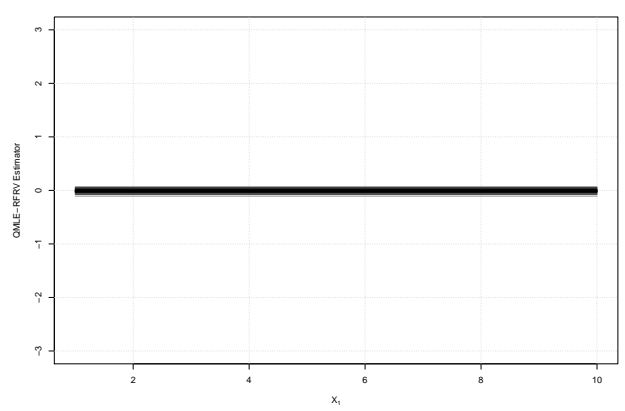

(c) QMLE-RFRV, D2 =0

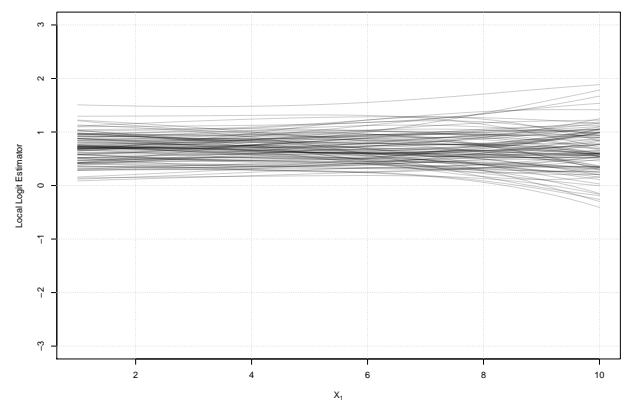

(b) Local logit regression, D2 = 1

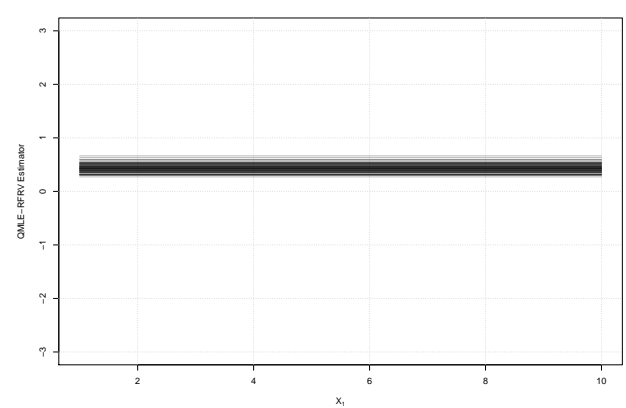

(d) QMLE-RFRV, D2 = 1

Figure 1: The interaction effects estimates of $x_{1}$ conditional on $d_{2}$ under simulation M1

Note: These figures show the interaction effect estimates of $x_{1}$ and $d_{2}$ for the simulation assumption M1. (a) and (b) illustrate the local logit marginal effect estimates $\beta_{1}(x)$ as a function of $x_{1}$ conditional on $d_{1}=0$ and 1 , respectively, as specified in (10). On the other hand, (c) and (d) represent the parametric QMLE-RFRV estimates $\gamma_{1}$ and $\gamma_{1}+\gamma_{6}$, respectively, as specified in (9). 


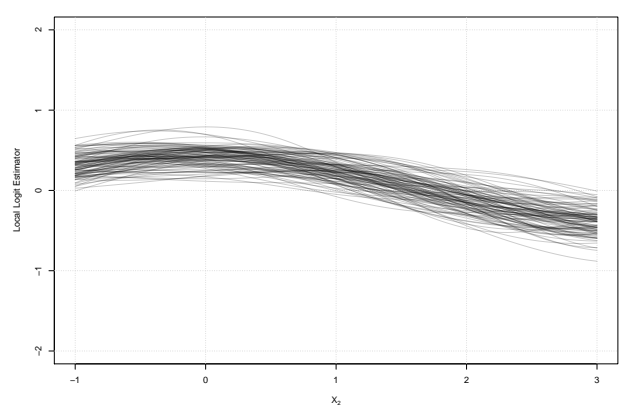

(a) Local logit regression

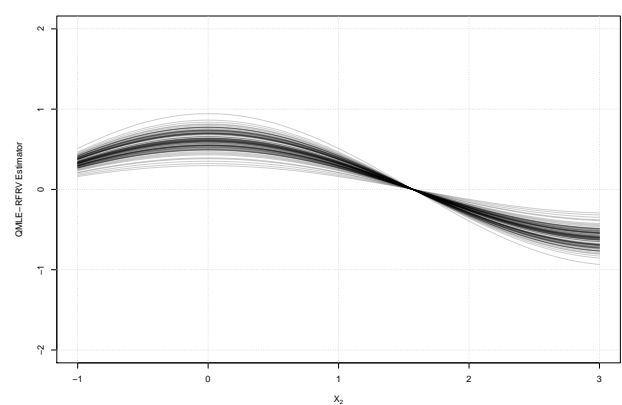

(b) QMLE-RFRV

Figure 2: The nonlinear marginal effect estimates of $x_{2}$ under simulation M1

Note: (a) is the local logit marginal effect estimate $\beta_{2}(x)$ in (10) as a function of $x_{2}$. (b) represents the parametric QMLE-RFRV estimate $\gamma_{2} \cos \left(x_{2}\right)$ as the marginal effect estimate of $x_{2}$ in (9).

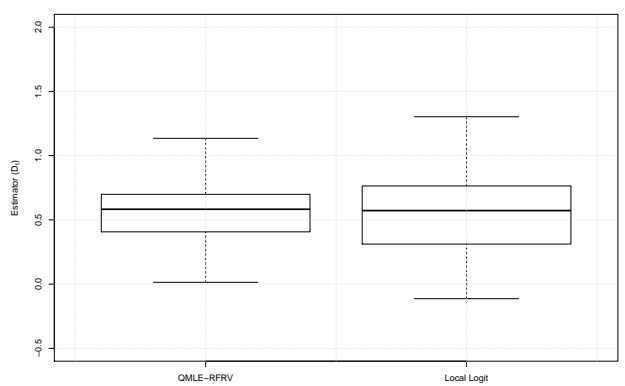

(a) $D_{1}$

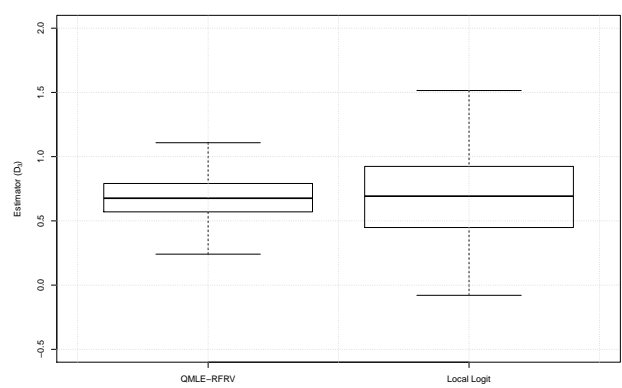

(b) $D_{3}$

Figure 3: The marginal effect estimates of $D_{1}$ and $D_{3}$ under simulation M1

Note: (a) and (b) compare the marginal effect estimates of QMLE-RFRV and the local logit model for the discrete variables $D_{1}$ and $D_{3}$ under simulation assumption M1 in (9) and (10). (a) represents the marginal effect estimates of $D_{1}$, which compares $\hat{\gamma}_{3}$ and $\hat{\beta}_{3}(x)$, on the left and right hand sides of the figure, respectively. Similarly, (b) represents the comparison of the marginal effect of $D_{3}$ between $\hat{\gamma}_{5}$ and $\hat{\beta}_{5}(x)$.

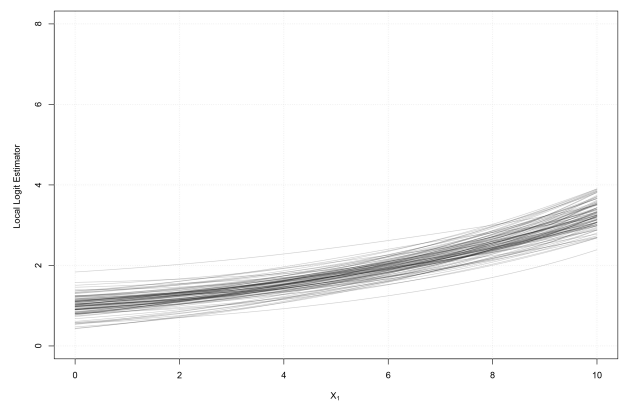

(a) Local logit regression

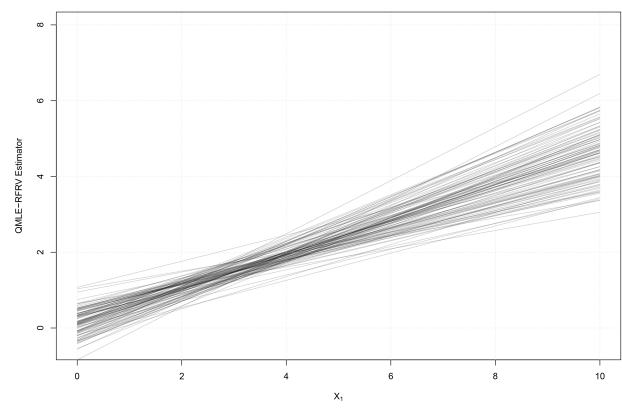

(b) QMLE-RFRV

Figure 4: The interaction effect estimates of $D_{2}$ conditional on $x_{1}$ under simulation M1

Note: These figures show the marginal effect estimate of $d_{2}$ as a function of $x_{1}$ as such interaction effect is specified in the simulation assumption M1. (a) illustrates the local logit marginal effect estimate $\beta_{4}(x)$ in (10) as a function of $x_{1}$. (b) is represents the marginal effect estimate $\gamma_{4}+\gamma_{6} x_{1}$ in (9). 


\section{Appendix B: Empirical results}

\section{Tables and figures}

\begin{tabular}{|c|c|c|c|c|c|c|c|c|}
\hline \multirow[b]{2}{*}{ Variables } & \multirow[b]{2}{*}{ Frequency } & \multirow[b]{2}{*}{$\%$} & \multicolumn{6}{|c|}{ Recovery rate at $\tau$-quantile } \\
\hline & & & Mean & $5 \%$ & $25 \%$ & $50 \%$ & $75 \%$ & $95 \%$ \\
\hline Recovery rate & 3,573 & $100 \%$ & 0.5570 & 0.0000 & 0.1849 & 0.5888 & 1.0000 & 1.0000 \\
\hline \multicolumn{9}{|l|}{ Panel A: Discrete Variables } \\
\hline \multicolumn{9}{|l|}{ (i) Type of loans } \\
\hline Term loans $\left(\right.$ Type $^{(1)}$ - reference category) & 746 & $21 \%$ & 0.7054 & 0.0222 & 0.4343 & 0.8638 & 1.0000 & 1.0000 \\
\hline Revolving loans $\left(\right.$ Type $\left.^{(2)}\right)$ & 738 & $21 \%$ & 0.8251 & 0.2147 & 0.6934 & 1.0000 & 1.0000 & 1.0000 \\
\hline Senior secured bonds $\left(\right.$ Type $\left.^{(3)}\right)$ & 446 & $12 \%$ & 0.5896 & 0.1079 & 0.2093 & 0.5682 & 1.0000 & 1.0000 \\
\hline Senior subordinated bonds $\left(\right.$ Type $\left.^{(4)}\right)$ & 355 & $10 \%$ & 0.2429 & 0.0000 & 0.0080 & 0.1005 & 0.3779 & 0.9225 \\
\hline Senior unsecure bonds $\left(\right.$ Type $\left.^{(5)}\right)$ & 1,061 & $30 \%$ & 0.4263 & 0.0000 & 0.1027 & 0.3578 & 0.7257 & 1.0000 \\
\hline Junior and subordinate bonds $\left(\right.$ Type $\left.{ }^{(6)}\right)$ & 227 & $6 \%$ & 0.2352 & 0.0000 & 0.0000 & 0.0968 & 0.3491 & 1.0000 \\
\hline \multicolumn{9}{|l|}{ (ii) Instrument rank } \\
\hline $\operatorname{Rank}^{(1)}$ (reference category) & 1,711 & $48 \%$ & 0.7476 & 0.1221 & 0.5151 & 1.0000 & 1.0000 & 1.0000 \\
\hline $\operatorname{Rank}^{(2)}$ & 1,258 & $35 \%$ & 0.4294 & 0.0000 & 0.1159 & 0.3170 & 0.7422 & 1.0000 \\
\hline $\operatorname{Rank}^{(3)}$ & 393 & $11 \%$ & 0.2994 & 0.0000 & 0.0041 & 0.1531 & 0.5255 & 1.0000 \\
\hline $\operatorname{Rank}^{(4)}$ & 211 & $6 \%$ & 0.2514 & 0.0000 & 0.0010 & 0.1027 & 0.3615 & 0.9122 \\
\hline \multicolumn{9}{|l|}{ (iii) Collateral } \\
\hline Uncollaterized loans (reference category) & 1,712 & $48 \%$ & 0.3685 & 0.0000 & 0.0396 & 0.2372 & 0.6638 & 1.0000 \\
\hline Collaterized loans & 1,861 & $52 \%$ & 0.7303 & 0.1240 & 0.4545 & 0.9622 & 1.0000 & 1.0000 \\
\hline \multicolumn{9}{|l|}{ Panel B: Continuous variables } \\
\hline \multicolumn{9}{|l|}{ (i) Debt Cushion (DC) } \\
\hline $\mathrm{DC}=0$ & 1,631 & $46 \%$ & 0.3969 & 0.0000 & 0.0738 & 0.2840 & 0.6959 & 1.0000 \\
\hline $0<\mathrm{DC}<0.5$ & 1,049 & $29 \%$ & 0.5345 & 0.0043 & 0.2092 & 0.5476 & 0.8961 & 1.0000 \\
\hline $0.5<\mathrm{DC}<1$ & 893 & $25 \%$ & 0.8766 & 0.2386 & 0.9435 & 1.0000 & 1.0000 & 1.0000 \\
\hline \multicolumn{9}{|l|}{ (ii) Stress index (SI) } \\
\hline $\mathrm{SI} \leq 0$ & 853 & $24 \%$ & 0.7058 & 0.0022 & 0.4728 & 0.8585 & 1.0000 & 1.0000 \\
\hline $0<\mathrm{SI}<1$ & 2,272 & $63 \%$ & 0.5102 & 0.0000 & 0.1533 & 0.4654 & 1.0000 & 1.0000 \\
\hline $\mathrm{SI} \geq 1$ & 448 & $12 \%$ & 0.5105 & 0.0000 & 0.1344 & 0.4617 & 1.0000 & 1.0000 \\
\hline
\end{tabular}

Table 5: Summary statistics and a contingency table of the empirical recovery rate data

Note: The full data is partitioned to several sub-samples by the given variables in the column 1 , then columns 2 and 3 report the number of the observation in each sub-sample and its percentage to the total observation, respectively. Columns 4 to 9 is a contingency table, which indicates the RR density of each sub-sample. 


\begin{tabular}{|c|c|c|}
\hline Variables & $\begin{array}{c}\text { Parametric } \\
\text { coefficients }(\gamma)\end{array}$ & $\begin{array}{c}\text { Median of } \\
\text { local logit coefficients }(\beta(x))\end{array}$ \\
\hline \multicolumn{3}{|l|}{ Type of loan } \\
\hline Type $e^{(2)}$ & $\begin{array}{c}0.4907^{* * *} \\
(0.1358)\end{array}$ & 0.4376 \\
\hline Type ${ }^{(3)}$ & $\begin{array}{l}-0.0229 \\
(0.1433)\end{array}$ & -0.0328 \\
\hline Type ${ }^{(4)}$ & $\begin{array}{l}-0.3771 \\
(0.2331)\end{array}$ & -0.3857 \\
\hline Type $e^{(5)}$ & $\begin{array}{c}0.2535 \\
(0.2010)\end{array}$ & 0.2899 \\
\hline Type $e^{(6)}$ & $\begin{array}{l}-0.4258 \\
(0.2476)\end{array}$ & -0.4417 \\
\hline \multicolumn{3}{|l|}{ Rank } \\
\hline Rank 2 & $\begin{array}{c}-0.4512^{* * *} \\
(0.1049)\end{array}$ & -0.5076 \\
\hline Rank 3 & $\begin{array}{c}-0.7900^{* * *} \\
(0.1506)\end{array}$ & -0.8919 \\
\hline Rank 4 & $\begin{array}{c}-0.9300^{* * *} \\
(0.1896)\end{array}$ & -1.0193 \\
\hline Collateral & & \\
\hline Collateralized loan & $\begin{array}{c}0.4420^{* *} \\
(0.1842)\end{array}$ & 0.6127 \\
\hline
\end{tabular}

Table 6: The estimates of the QMLE-RFRV model and the local logit regression

Note: The median of the local estimates are calculated based on the results in Figure 9 


\begin{tabular}{|c|c|c|c|}
\hline \multirow{2}{*}{ Variables } & \multirow{2}{*}{ LL estimates } & \multicolumn{2}{|c|}{ Sample averages RR } \\
\hline & & Category of interest & Reference category \\
\hline
\end{tabular}

Panel A Senior secured bond

$\begin{array}{lccc}D C \in[0,1] & \mathrm{N} / \mathrm{A} & 0.59 & 0.71 \\ D C<0.2 & \text { Negative } & 0.44 & 0.53 \\ 0.2 \leq D C<0.6 & \text { Positive } & 0.82 & 0.71 \\ D C \geq 0.6 & \text { Negative } & 0.79 & 0.91\end{array}$

Panel B Senior unsecured bond

$\begin{array}{lccc}D C \in[0,1] & \text { N } / \mathrm{A} & 0.43 & 0.71 \\ D C<0.2 & \text { Positive } & 0.41 & 0.34 \\ 0.2 \leq D C<0.5 & \text { Negative } & 0.47 & 0.74 \\ D C \geq 0.5 & \text { Positive } & 0.77 & 0.36\end{array}$

Panel C Loans with collateral

\begin{tabular}{lccc}
$D C \in[0,1]$ & $\mathrm{N} / \mathrm{A}$ & 0.73 & 0.37 \\
$D C<0.2$ & Positive & 0.53 & 0.31 \\
$0.2 \leq D C<0.5$ & Negative & 0.68 & 0.63 \\
$D C \geq 0.5$ & Positive & 0.92 & 0.65 \\
\hline
\end{tabular}

Table 7: The average recovery rates of senior bonds and collateralized loans for various ranges of DC

Note: The empirical RR is partitioned based on the findings in the interaction analysis of local logit model estimates of senior bonds and collateral status. Then RR of each sub-sample is compared with the reference group. For Panels A and $\mathrm{B}$, given the ranges of $\mathrm{DC}$ in the first column, the categories of interest are the senior secured and unsecured bonds, respectively, and the reference categories are collateralized and uncollateralized term loans, respectively. For Panel $\mathrm{C}$, the the category of interest is the collateralized loan, whereas the reference category is the uncollateralized loan. 


\begin{tabular}{|c|c|c|c|c|}
\hline Coefficients & Variables & Estimates & (SE) & \\
\hline \multicolumn{5}{|c|}{ Panel A: Debt cushion } \\
\hline$\gamma_{D C_{1}}$ & $I(D C<0.6) D C^{2}$ & 5.5071 & $(0.5890)$ & $*$ \\
\hline$\gamma_{D C_{2}}$ & $I(D C \geq 0.6) D C$ & 2.4200 & $(0.2105)$ & $*$ \\
\hline \multicolumn{5}{|c|}{ Panel B: Stress index } \\
\hline$\gamma_{S I_{1}}$ & $I(S I<0) S I$ & -1.2283 & $(0.1436)$ & $*$ \\
\hline$\gamma_{S I_{2}}$ & $I(0 \leq S I<1.5) S I$ & -0.4589 & $(0.0997)$ & $*$ \\
\hline$\gamma_{S_{I_{3}}}$ & $I(S I \geq 1.5) S I$ & -0.0212 & $(0.0330)$ & \\
\hline \multicolumn{5}{|c|}{ Panel C: Types of loan } \\
\hline$\gamma_{T_{21}}$ & Type $e^{(2)}$ & 0.0817 & $(0.1124)$ & \\
\hline$\gamma_{T_{22}}$ & $I(D C>0.8)$ Type $e^{(2)}$ & 0.2770 & $(0.5021)$ & \\
\hline$\gamma_{T_{31}}$ & $I(D C<0.2)$ Type $e^{(3)}$ & -0.4516 & $(0.1912)$ & * \\
\hline$\gamma_{T_{32}}$ & $I(0.2 \leq D C<0.6)$ Type $^{(3)}$ & 0.8757 & $(0.1883)$ & * \\
\hline$\gamma_{T_{33}}$ & $I(D C \geq 0.6)$ Type $e^{(3)}$ & -1.5847 & $(0.4087)$ & * \\
\hline$\gamma_{T_{41}}$ & Type $e^{(4)}$ & -0.3635 & $(0.1265)$ & * \\
\hline$\gamma_{T_{51}}$ & $I(D C<0.2)$ Type $^{(5)}$ & 0.2713 & $(0.0574)$ & $*$ \\
\hline$\gamma_{T_{52}}$ & $I(0.2 \leq D C<0.5)$ Type $^{(5)}$ & -0.0456 & $(0.1806)$ & \\
\hline$\gamma_{T_{53}}$ & $I(D C \geq 0.5)$ Type $^{(5)}$ & 0.8014 & $(0.4795)$ & \\
\hline$\gamma_{T_{61}}$ & Type $e^{(6)}$ & -0.3691 & $(0.1431)$ & $*$ \\
\hline \multicolumn{5}{|c|}{ Panel D: Collateral status } \\
\hline$\gamma_{C_{1}}$ & $I(D C<0.2) C o l$ & 0.5802 & $(0.1733)$ & $*$ \\
\hline$\gamma_{C_{2}}$ & $I(0.2 \leq D C<0.5) \mathrm{Col}$ & -0.1600 & $(0.1383)$ & \\
\hline$\gamma_{C_{3}}$ & $I(D C \geq 0.5) C o l$ & 1.2458 & $(0.3536)$ & * \\
\hline \multicolumn{5}{|c|}{ Panel E: Instrumental rank } \\
\hline$\gamma_{R_{2}}$ & Rank2 & -1.0040 & $(0.0901)$ & * \\
\hline$\gamma_{R_{3}}$ & Rank3 & -1.5447 & $(0.1204)$ & $*$ \\
\hline$\gamma_{R_{4}}$ & Rank4 & -1.6792 & $(0.1422)$ & $*$ \\
\hline
\end{tabular}

Table 8: Estimates of the calibrated parametric QMLE-RFRV model

Note: The calibrated model $(\mathrm{CM})$ parameters and the corresponding variables are presented in columns $1 \& 2$ respectively. Panels A, B, C, D and E list nonlinear, interactive, discretised DC and discrete variables in the model 


\begin{tabular}{|c|c|c|c|c|c|c|c|c|}
\hline \multicolumn{3}{|c|}{ Out-of-sample } & \multicolumn{3}{|c|}{ MSE } & \multicolumn{3}{|c|}{ MAE } \\
\hline Year & Out-of-time obs. & $\%$ & Benchmark & LL & $\mathrm{CM}$ & Benchmark & LL & $\mathrm{CM}$ \\
\hline 2001-2012 & 2,738 & $77 \%$ & 0.1071 & 0.1048 & 0.1203 & 0.2449 & 0.2425 & 0.2505 \\
\hline 2002-2012 & 2,168 & $61 \%$ & 0.1035 & 0.1052 & 0.1229 & 0.2420 & 0.2445 & 0.2508 \\
\hline 2003-2012 & 1,478 & $41 \%$ & 0.1021 & 0.1007 & 0.1411 & 0.2608 & 0.2557 & 0.2672 \\
\hline 2004-2012 & 1,141 & $32 \%$ & 0.1131 & 0.0900 & 0.1017 & 0.2536 & 0.2238 & 0.2284 \\
\hline 2005-2012 & 965 & $27 \%$ & 0.1279 & 0.1182 & 0.1053 & 0.2689 & 0.2563 & 0.2410 \\
\hline 2006-2012 & 779 & $22 \%$ & 0.1439 & 0.1045 & 0.1179 & 0.2843 & 0.2464 & 0.2556 \\
\hline 2007-2012 & 708 & $20 \%$ & 0.1517 & 0.1145 & 0.1219 & 0.2932 & 0.2578 & 0.2613 \\
\hline 2008-2012 & 660 & $18 \%$ & 0.1592 & 0.1144 & 0.1245 & 0.3016 & 0.2570 & 0.2647 \\
\hline 2009-2012 & 496 & $14 \%$ & 0.0841 & 0.0825 & 0.0831 & 0.2288 & 0.2170 & 0.2273 \\
\hline 2010-2012 & 121 & $3 \%$ & 0.0643 & 0.0825 & 0.0746 & 0.2024 & 0.2065 & 0.2004 \\
\hline 2011-2012 & 35 & $1 \%$ & 0.0709 & 0.0819 & 0.0954 & 0.2092 & 0.2055 & 0.2234 \\
\hline & Average & & 0.1116 & 0.0999 & 0.1099 & 0.2536 & 0.2375 & 0.2428 \\
\hline & Var. & & 0.0009 & 0.0002 & 0.0004 & 0.0010 & 0.0004 & 0.0004 \\
\hline
\end{tabular}

Table 9: Out-of-sample predictive performance of models

Note: The table employs the data partitioning (DF2). Columns 1-3 indicate the out-of-sample period, the number of the observation in out-sample period, and the percentage of these observations relative to total number. Benchmark, LL and CM represent the standard QMLE-RFRV model, local logit model and the calibrated QMLE-RFRV model, respectively.

\begin{tabular}{|c|c|c|c|c|c|c|c|c|}
\hline \multirow{2}{*}{$\begin{array}{c}\text { Out-of-sample } \\
\text { year }\end{array}$} & \multicolumn{2}{|c|}{ Sample size } & \multicolumn{3}{|c|}{ MSE } & \multicolumn{3}{|c|}{ MAE } \\
\hline & Out-of-sample & In-sample & Benchmark & LL & $\mathrm{CM}$ & Benchmark & LL & $\mathrm{CM}$ \\
\hline 2001 & 570 & 3003 & 0.1308 & 0.1235 & 0.1274 & 0.2878 & 0.2826 & 0.2860 \\
\hline 2002 & 690 & 2883 & 0.0903 & 0.0861 & 0.0779 & 0.2088 & 0.2102 & 0.2175 \\
\hline 2003 & 337 & 3236 & 0.1045 & 0.0987 & 0.1049 & 0.2814 & 0.2757 & 0.2533 \\
\hline 2004 & 176 & 3397 & 0.0690 & 0.0555 & 0.0692 & 0.1828 & 0.1678 & 0.1786 \\
\hline 2005 & 186 & 3387 & 0.1114 & 0.0815 & 0.0776 & 0.2245 & 0.2000 & 0.1959 \\
\hline 2006 & 71 & 3502 & 0.0950 & 0.0708 & 0.0782 & 0.2067 & 0.1932 & 0.1947 \\
\hline 2007 & 48 & 3525 & 0.0919 & 0.0739 & 0.0756 & 0.1959 & 0.1924 & 0.2078 \\
\hline 2008 & 164 & 3409 & 0.1322 & 0.0937 & 0.1082 & 0.3454 & 0.2911 & 0.3127 \\
\hline 2009 & 375 & 3198 & 0.0903 & 0.0860 & 0.0911 & 0.2373 & 0.2215 & 0.2365 \\
\hline 2010 & 86 & 3487 & 0.0615 & 0.0728 & 0.0623 & 0.1996 & 0.2028 & 0.1901 \\
\hline 2011 & 20 & 3553 & 0.0838 & 0.1183 & 0.1001 & 0.2210 & 0.2299 & 0.2510 \\
\hline & Average & & 0.0964 & 0.0873 & 0.0884 & 0.2356 & 0.2243 & 0.2295 \\
\hline & Var. & & 0.0018 & 0.0007 & 0.0011 & 0.0022 & 0.0015 & 0.0017 \\
\hline
\end{tabular}

Table 10: Out-of-sample predictive performance of the models over the course of economic cycle

Note: The table employs the data partitioning (DF3). The model is estimated for the in-sample period, which excludes only one year - the out-of-sample year. 


\begin{tabular}{ccccc}
\hline Quantiles & Actual & Benchmark & LL & CM \\
\hline \multirow{2}{*}{0.05} & 57.71 & 59.68 & 56.93 & 56.11 \\
& (\% different) & $(3.4 \%)$ & $(1.4 \%)$ & $(2.9 \%)$ \\
\multirow{2}{*}{0.25} & 61.40 & 64.54 & 59.80 & 59.25 \\
& (\% different) & $(5.1 \%)$ & $(2.7 \%)$ & $(3.6 \%)$ \\
\multirow{2}{*}{0.5} & 63.69 & 67.91 & 61.35 & 61.28 \\
& (\% different) & $(6.6 \%)$ & $(3.8 \%)$ & $(3.9 \%)$ \\
0.75 & 65.91 & 70.55 & 62.99 & 62.97 \\
\multirow{2}{*}{0.95} & (\% different) & $(7.0 \%)$ & $(4.6 \%)$ & $(4.7 \%)$ \\
& 69.19 & 74.94 & 65.55 & 65.99 \\
& $(\%$ different) & $(8.3 \%)$ & $(5.6 \%)$ & $(4.8 \%)$ \\
\hline & MSE & 22.09 & 16.52 & 15.72 \\
\hline
\end{tabular}

Table 11: Quantile predictive performance of the models

Note: Portfolio distributions were generated from the out-of-sample predictions of RR by the three models.

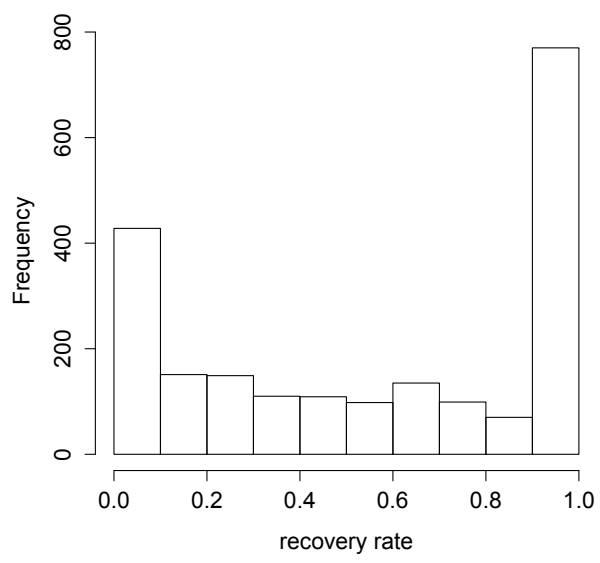

Figure 5: The empirical density of the recovery rate 


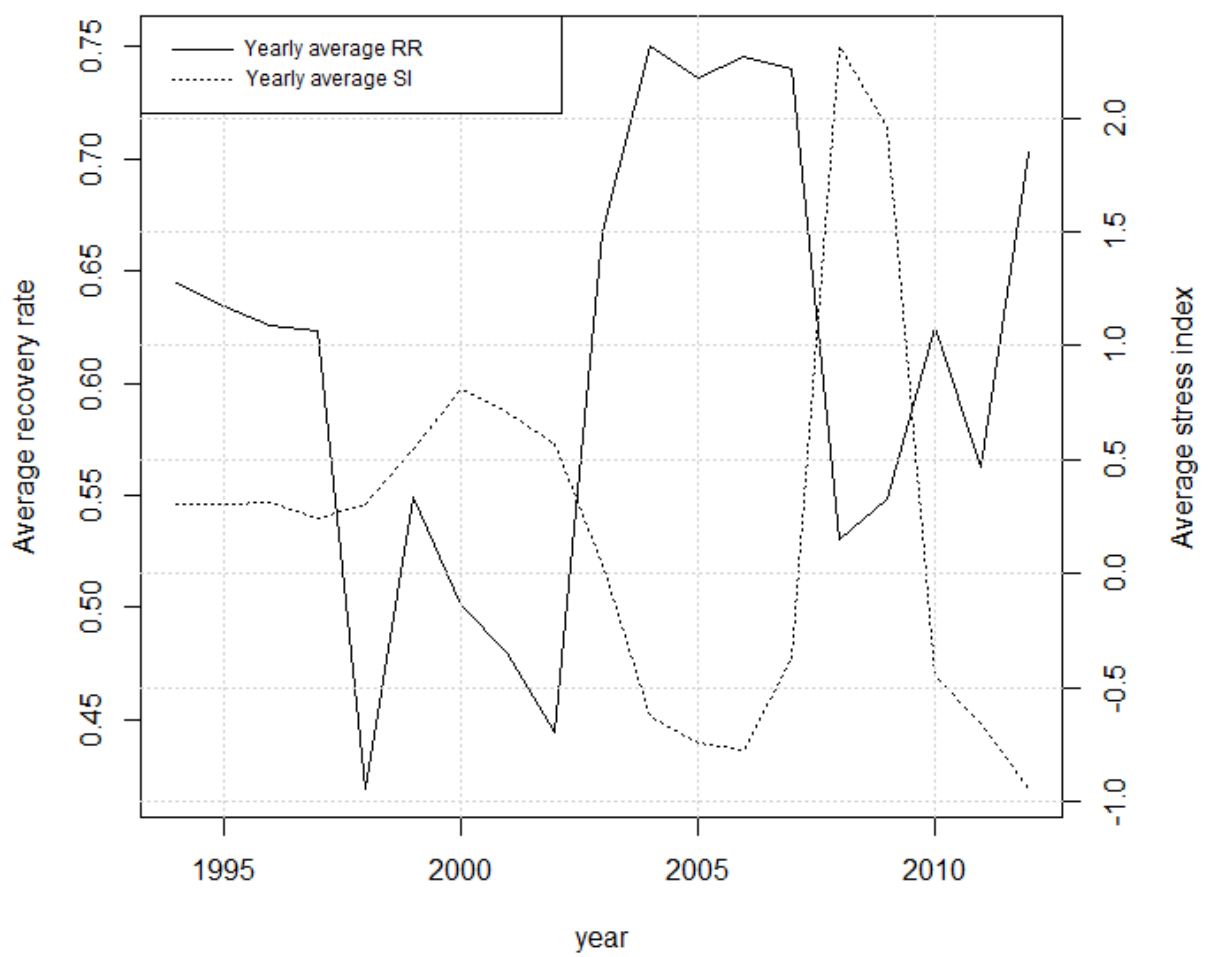

Figure 6: The annual averages of stress index and recovery rate from 1994 to 2013

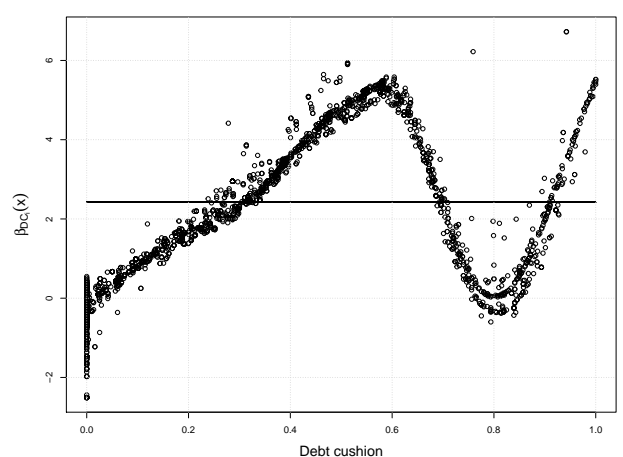

(a) Debt cushion

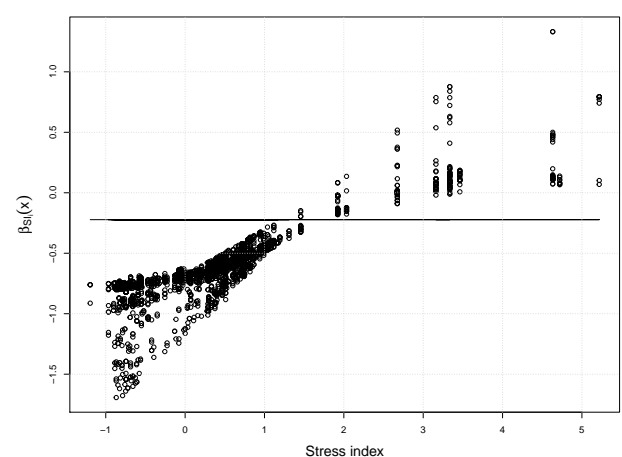

(b) Stress index

Figure 7: The local estimator of the debt cushion and the stress index

Note: (a) is the nonlinear local logit marginal effect estimate of DC, $\hat{\beta}_{D C}(x)$, as a function of $D C$. (b) illustrates the marginal effect estimate of SI, $\hat{\beta}_{S I}(x)$, as a function of SI. In addition, the dark solid lines in both figures are the parametric coefficients of QMLE-RFRV for DC and SI. 


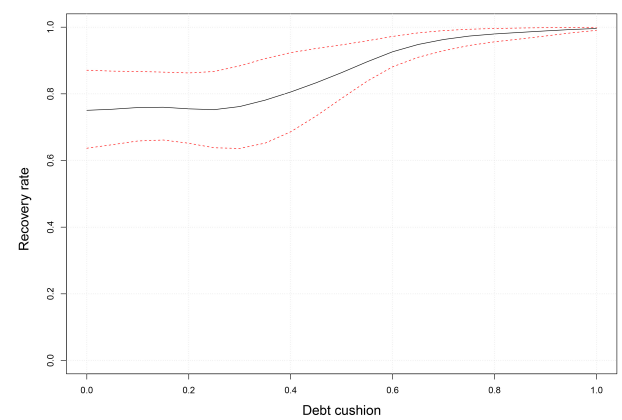

Figure 8: The effect of debt cushion on the recovery rate of a specific defaulted loan

Note: The figure illustrates the effect of debt cushion on the recovery rate of a collateralised revolving loan with rank 1 (Type $=2$, Rank $=1, \mathrm{Col}=1)$ as a defined specific characteristics. We also consider the effect in three different economic scenarios by specifying the levels of the stress index. The dark solid line represents the effect of debt cushion on the recovery rate given SI = 0, while the red dashed lines represent the effects during SI $=-1$ for the lower bound, and SI $=1$ for the upper bound.

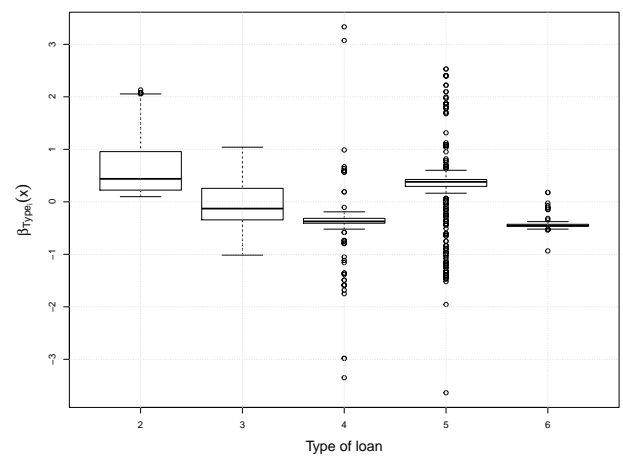

(a) Types of loan

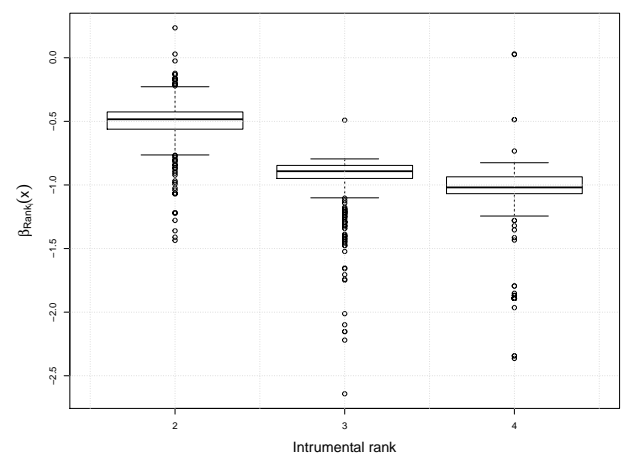

(b) Instrumental rank

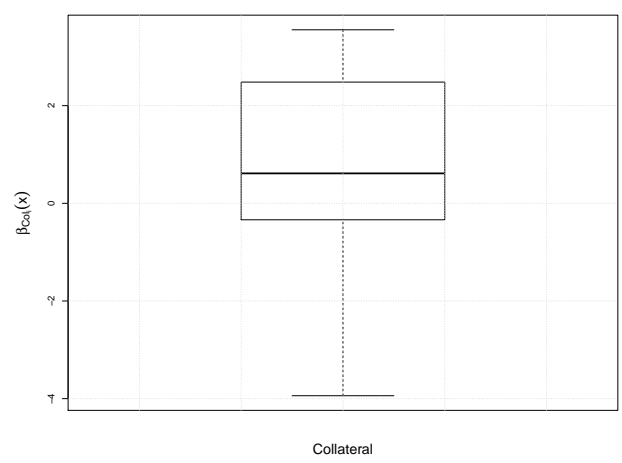

(c) Collateral status

Figure 9: The marginal effects of the categorical RR-covariates

Note: The figures represent the marginal effect of each categorical covariate: (a) shows the marginal effects of 5 types of loan, Type $=\{2, . ., 6\}$, where the term loan (Type $=1$ ) is the reference category; (b) shows the marginal effects of three instrumental ranks, Rank $=\{2,3,4\}$, where Rank 1 is the reference category; and (c) is the marginal effect of the collateral status, $\mathrm{Col}=1$, where uncollateralized loan is the reference category. 


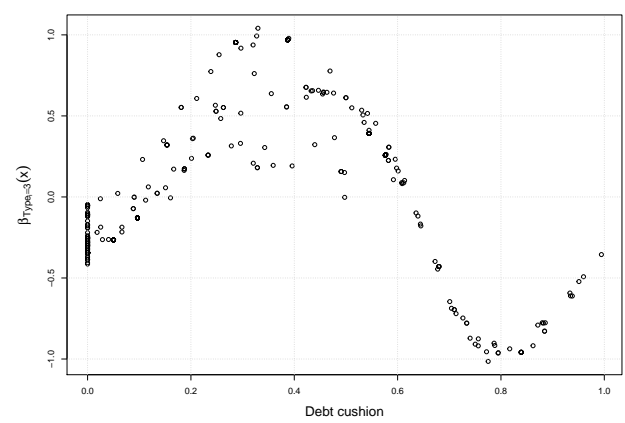

(a) Senior secured bond (Type $=3$ )

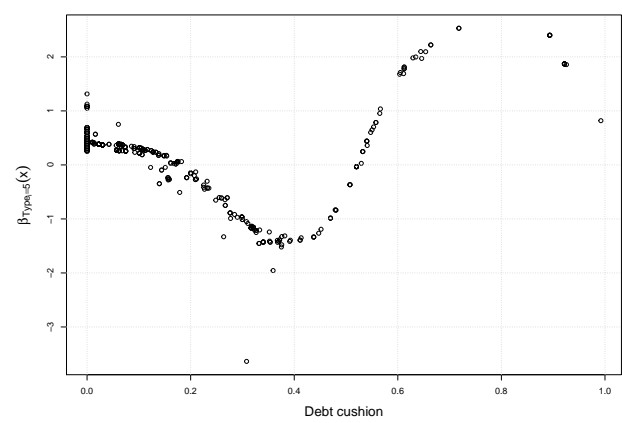

(b) Senior unsecured bond (Type $=5)$

Figure 10: The interaction effect estimates of the senior bonds conditional on level of the debt cushion

Note: The figures illustrate the local logit marginal effect estimates of Type $=\{3,5\}$, which are $\hat{\beta}_{\text {Type }}^{(3)}(x)$ and $\hat{\beta}_{\text {Type }}{ }^{(5)}(x)$, respectively, as a function of the debt cushion

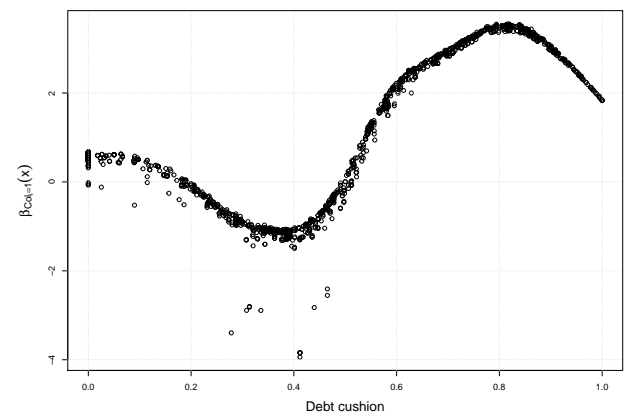

Figure 11: The interaction effect estimate of the collateral status conditional on level of the debt cushion

Note: The figure illustrates the local logit marginal effect estimates of the collateralized loan, which is $\hat{\beta}_{C o l}(x)$, as a function of the debt cushion

\section{References}

Altman, E. I., \& Kalotay, E. A. (2014). Ultimate recovery mixtures. Journal of Banking E) Finance, 40, 116-129.

Bastos, J. A. (2010). Forecasting bank loans loss-given-default. Journal of Banking \& Finance, 34, 2510-2517.

BIS. (2004). International convergence of capital measurement and capital standards: A revised framework. Bank for International Settlements.

Calabrese, R., \& Zenga, M. (2010). Bank loan recovery rates: Measuring and nonparametric density estimation. Journal of Banking \& Finance, 34, 903-911.

Dermine, J., \& De Carvalho, C. N. (2006). Bank loan losses-given-default: A case study. Journal of Banking $\&$ Finance, 30, 1219-1243. 
De Servigny, A., Renault, O., \& de Servigny, A. (2004). Measuring and managing credit risk.

Fan, J., Zhang, C., \& Zhang, J. (2001). Generalized likelihood ratio statistics and wilks phenomenon. Annals of statistics, 153-193.

Frölich, M. (2006). Non-parametric regression for binary dependent variables. The Econometrics Journal, 9(3), 511-540.

Gupton, G., \& Stein, R. (2005). Losscalc v2: Dynamic prediction of losses-given-default modeling methodology. Moody's KMV.

Kalotay, E. A., \& Altman, E. I. (2016). Intertemporal forecasts of defaulted bond recoveries and portfolio losses. Review of Finance, rfw028.

Khieu, H. D., Mullineaux, D. J., \& Yi, H.-C. (2012). The determinants of bank loan recovery rates. Journal of Banking \& Finance, 36, 923-933.

Kliesen, K. L., Smith, D. C., et al. (2010). Measuring financial market stress. Economic Synopses.

Loterman, G., Brown, I., Martens, D., Mues, C., \& Baesens, B. (2012). Benchmarking regression algorithms for loss given default modeling. International Journal of Forecasting, 28, 161-170.

Papke, L. E., \& Wooldridge, J. M. (1996). Econometric methods for fractional response variables with an application to 401 (k) plan participation rates. Journal of Applied Econometrics, 11(6), 619-632.

Qi, M., \& Yang, X. (2009). Loss given default of high loan-to-value residential mortgages. Journal of Banking \& Finance, 33, 788-799.

Qi, M., \& Zhao, X. (2011). Comparison of modeling methods for loss given default. Journal of Banking \& Finance, 35, 2842-2855.

Racine, J., \& Li, Q. (2004). Nonparametric estimation of regression functions with both categorical and continuous data. Journal of Econometrics, 119(1), 99-130.

Schuermann, T. (2004). What do we know about loss given default?

Siao, J.-S., Hwang, R.-C., \& Chu, C.-K. (2015). Predicting recovery rates using logistic quantile regression with bounded outcomes. Quantitative Finance, 16, 1-16.

Sopitpongstorn, N., Gao, J., Silvapulle, P., \& Zhang, X. (2014). Non- and semi- parametric conditional mean regressions for recovery rate. Monash Econometric and Business 
Statistics seminar 2014.

Sopitpongstorn, N., Gao, J., Silvapulle, P., \& Zhang, X. (2016). Non-and semi-parametric quantile models for recovery rate. 29th Australasian Finance and Banking Conference 2016.

Tong, E. N., Mues, C., \& Thomas, L. (2013). A zero-adjusted gamma model for mortgage loan loss given default. International Journal of Forecasting, 29, 548-562.

Van de Castle, K., \& Keisman, D. (1999). Recovering your money: Insights into losses from defaults. Standard \& Poor's Credit Week, 16, 1999.

Yao, X., Crook, J., \& Andreeva, G. (2015). Support vector regression for loss given default modelling. European Journal of Operational Research, 240, 528-538. 